University of Nebraska - Lincoln

DigitalCommons@University of Nebraska - Lincoln

May 1976

\title{
Photoinization cross sections for atomic chlorine using an open- shell random-phase approximation
}

Anthony F. Starace

University of Nebraska-Lincoln, astarace1@unl.edu

Lloyd Armstrong Jr.

Johns Hopkins University, Baltimore, Maryland

Follow this and additional works at: https://digitalcommons.unl.edu/physicsstarace

Part of the Physics Commons

Starace, Anthony F. and Armstrong, Lloyd Jr., "Photoinization cross sections for atomic chlorine using an open-shell random-phase approximation" (1976). Anthony F. Starace Publications. 7.

https://digitalcommons.unl.edu/physicsstarace/7

This Article is brought to you for free and open access by the Research Papers in Physics and Astronomy at DigitalCommons@University of Nebraska - Lincoln. It has been accepted for inclusion in Anthony F. Starace Publications by an authorized administrator of DigitalCommons@University of Nebraska - Lincoln. 


\title{
Photoionization cross sections for atomic chlorine using an open-shell random-phase approximation
}

\author{
Anthony F. Starace* ${ }^{\dagger}$ \\ Behlen Laboratory of Physics, The University of Nebraska, Lincoln, Nebraska 68588 \\ Lloyd Armstrong, Jr. ${ }^{\ddagger}$ \\ Physics Department, Johns Hopkins University, Baltimore, Maryland 21218
}

(Received 3 November 1975)

\begin{abstract}
The use of the random-phase approximation with exchange (RPAE) for calculating partial and total photoionization cross sections and photoelectron angular distributions for open-shell atoms is examined for atomic chlorine. Whereas the RPAE corrections in argon $(Z=18)$ are large, we find those in chlorine ( $Z$ $=17$ ) to be much smaller due to geometric factors. Hartree-Fock calculations with and without core relaxation are also presented. Sizable deviations from the close-coupling results of Conneely are found.
\end{abstract}

\section{INTRODUCTION}

If one excludes the lightest elements (i.e., $1 \leqslant Z \leqslant 10$ ), then it is fair to say that the role of electron correlations in atomic photoionization processes has been extensively studied only for the rare gases and a few other closed-shell systems. The reasons for this are, firstly, the availability of detailed experimental rare-gas photoabsorption spectra, ${ }^{1}$ and secondly, the relative theoretical ease of dealing with spherically symmetric targets having only a few finalstate channels. The key to the current theoretical understanding of these closed-shell photoabsorption spectra has been the random-phase approximation with exchange (RPAE). The RPAE calculations of Amus'ya, Cherepkov, and collaborators, ${ }^{2}$ and of Wendin, ${ }^{3}$ Lin, ${ }^{4}$ and Starace ${ }^{5}$ have amply demonstrated the important influence that virtual excitations of pairs of valence electrons have on closed-shell photoabsorption spectra and the necessity of taking these electron correlations into account in order to obtain good agreement with experiment.

Consider specifically the history of some of the theoretical work concerning argon, one of the most thoroughly studied atoms. Hartree-Fock, ${ }^{6,7}$ intrachannel, ${ }^{8}$ and close-coupling calculations ${ }^{9}$ failed to reproduce its experimental photoionization cross section. ${ }^{10}$ Not until the RPAE calculation of Amus'ya et al. ${ }^{2}$ was good agreement $(\simeq 10 \%)$ between theory and experiment obtained. Furthermore, the importance of the electron correlations that the RPAE includes has been confirmed in argon by the very accurate manybody perturbation-theory calculation of the cross section by Kelly and Simons. ${ }^{11}$ In addition, Burke and Taylor $^{12}$ have recently done an $R$-matrix calculation of the argon photoionization cross sec- tion that gives agreement with experiment comparable to that of the RPAE calculations. Thus, while for very heavy closed-shell atoms it has been found necessary to take into account core relaxation effects in addition to the electron correlations included in the RPAE, ${ }^{13}$ for the medium-heavy atom argon $(Z=18)$ the RPAE has given calculated cross sections that are in very good agreement with both many-body perturbation theory ${ }^{11}$ and $R$-matrix ${ }^{12}$ calculations and with experiment. ${ }^{10}$

Despite its success, the RPAE as originally developed for atomic photoabsorption by Altick and Glassgold, ${ }^{14}$ and as used subsequently, ${ }^{2-5}$ has been largely limited to the theoretical treatment of closed-shell atoms. The only exceptions have been an extension of the RPAE by Cherepkov et $a l .{ }^{15}$ to the treatment of photoionization of open-shell atoms having a half-filled valence shell (e.g., atomic nitrogen), and an extension of the RPAE by Dalgaard ${ }^{16}$ to the calculation of oscillator strengths for discrete transitions in openshell atoms having either two electrons or two vacancies in the valence shell (e.g., silicon). Very recently, however, the general case has been solved and there are now two RPAE theories suitable for calculating the photoionization cross section of an arbitrary open-shell atom; these are the theories of Armstrong ${ }^{17}$ and of Rowe and Ngo-Trong. ${ }^{18}$ While these two theories differ in the requirements they place on the excitation operators that induce transitions between initial and final states, they are otherwise very similar.

In this paper we use the open-shell RPAE theory of Armstrong ${ }^{17}$ for the first time to explore electron correlation effects on the photoionization cross sections and photoelectron angular distributions of atomic chlorine. We have chosen to study atomic chlorine $(Z=17)$, since it is adjacent 
to argon $(Z=18)$ in the periodic table and therefore might be expected to have similarly strong electron correlations that, as with argon, are accounted for adequately by the RPAE. In addition, it has been suggested ${ }^{19}$ that atomic chlorine, as well as the other halogen atoms, might in the near future be more readily studied experimentally than other open-shell atoms having $Z>10$. As further motivation for our study, we note again that at present the photoabsorption properties of approximately $75 \%$ of the elements in the periodic table, mostly open-shell atoms, are largely unstudied either experimentally ${ }^{1}$ or theoretically (except in the independent-electron approximation). ${ }^{20(a), 21}$

Section II describes the reduction of Armstrong's ${ }^{17}$ equations of motion to a form suitable for numerical computation. The computation itself is described in detail. In brief, we have employed a basis set of discrete and continuum wave functions computed in the Hartree-Fock (HF) approximation and we have ignored coupling between photoelectron channels. Section III presents our HF and RPAE calculations of total and partial photoionization cross sections and photoelectron angular distributions. We find that in contrast to argon, the electron correlations included in the RPAE have a weak effect on photoabsorption in atomic chlorine. We expect this result to hold even if future calculations include coupling between photoelectron channels. Comparison is made with a close-coupling calculation of Conneely. ${ }^{22,23}$ Section IV summarizes our results and conclusions concerning electron correlations in atomic chlorine and other halogen atoms.

\section{SOLUTION OF THE OPEN-SHELL RPAE EQUATIONS}

The equations-of-motion derivation ${ }^{24-26}$ of the RPAE starts by assuming a particular expansion for an excitation operator that induces transitions from the ground state (e.g., for photoionization this is the electric dipole operator). By taking appropriate commutators of this operator with the Hamiltonian of the atom, one obtains equations for the coefficients in the expansion of the excitation operator. These coefficients are all one needs to obtain numerical values for matrix elements of the excitation operator and hence for cross sections.

The Armstrong ${ }^{17}$ open-shell RPAE equations for the coefficient vectors $X$ and $Y$ of odd-parity single-electron excitation operators are given most simply in matrix form:

$$
\left(\begin{array}{cc}
A & D \\
D^{\dagger} & A^{*}
\end{array}\right)\left(\begin{array}{l}
X \\
Y
\end{array}\right)=\omega\left(\begin{array}{cc}
U & 0 \\
0 & -U^{*}
\end{array}\right)\left(\begin{array}{l}
X \\
Y
\end{array}\right) .
$$

The matrix $A$ represents the interaction between singly-excited final-state configurations, while the matrix $D$ represents the interaction between the ground state and doubly-excited states. The matrix $U$ distinguishes Eq. (1) from the analogous equation for the closed-shell RPAE for which $U$ becomes the unit matrix. $U$ differs from the unit matrix because of the multiplicity of parent states that an open-shell term level has. Finally, the excitation energy is labelled $\omega$, which equals the photon energy in a photoionization interaction.

General expressions for the matrices $A, D$, and $U$ in $L S$ coupling are given by Armstrong ${ }^{17}$ in terms of an arbitrary basis of independent particle wave functions. While these general expressions appear to be quite complex, they simplify considerably upon making a few restrictions. In this paper we make the following restrictions:

(i) We consider excitations from the open subshell only. The experience gained from using the RPAE for closed-shell atoms ${ }^{27}$ seems to indicate that intersubshell interactions are important only for subshells having a small photoionization cross section relative to the cross section for nearby subshells. Neglecting interactions between subshells serves to make $U$ diagonal and proportional to the square of a fractional parentage coefficient for the ground-state, open-subshell configuration.

(ii) We choose for the excited orbitals an independent particle basis set of restricted $\mathrm{HF}$ wave functions calculated (in $L S$ coupling) in the field of the $V^{N-1}$ potential $^{28}$ constructed from the set of $\mathrm{HF}$ discrete orbitals for the atom minus one of the open-subshell orbitals. In this calculation the spin and orbital angular momenta for the ion and for the total system are specified and a different calculation is carried out for each allowed channel defined by these quantum numbers. This choice of basis set simplifies the matrix $A$ by eliminating direct intrachannel interactions between continuum orbitals. $^{2}$

(iii) We ignore any interaction between different final-state channels, i.e., interchannel coupling. To define these channels in $L S$ coupling, consider the photoionization of the lowest term level ${ }^{2 S_{0}+1} L_{0}$ of the open subshell $n l_{0}^{N}$ of an atom $Q$ by means of the electric dipole interaction,

$$
\begin{aligned}
& \mathcal{Q}\left(n l_{0}^{N}\left({ }^{2} s_{0}+1 L_{0}\right)\right)+\hbar \omega \\
& \rightarrow \mathbb{Q}^{+}\left(n l_{0}^{N-1}\left({ }^{2 S_{c}+1} L_{c}\right)\right)+e^{-}\left(\epsilon, l\left({ }^{2 S_{0}+1} L\right)\right) .
\end{aligned}
$$

We label the final state, on the right-hand side in (2), by $i \epsilon$, where $i$, the channel index, is meant to specify particular values of $S_{c}$ and $L_{c}$ (the spin and orbital angular momentum of the ionic core), 
of $l$ (the photoelectron's orbital angular momentum), and of $L$ (the total orbital angular momentum of the final state). $\epsilon$ is the photoelectron energy, which may have discrete (i.e., negative) as well as continuum values. The electrostatic interaction is diagonal in $L$ and hence neglect of interchannel interactions amounts to neglecting matrix elements between states $i \epsilon$ and $j \epsilon^{\prime}$ having differing ionic-core terms ${ }^{2 S_{c}+1} L_{c}$ and/or differing photoelectron orbital momenta $l$. Closecoupling calculations indicate that these interactions do not have a large effect on the total cross section except in resonance regions. ${ }^{29} \mathrm{We}$ shall discuss our neglect of the interchannel interactions in more detail in Sec. III. Neglect of these interactions serves to make the matrix $A$ diagonal in $i$ and $\epsilon$ and equal to the product of the photon energy $\omega$ and the diagonal matrix $U$.

Expressions for matrix elements of $U, A$, and $D$ appropriate to restrictions (i) and (ii) above are derived in Appendix A starting from the general expressions for these matrices given by Eqs. (27), (28), and (30) of Ref. 17. Restriction (iii) implies that we keep only those matrix elements, given in Eqs. (A10), (A12), and (A17) in Appendix $\mathrm{A}$, that are diagonal in the channel indices $i$ and $j$. In order to indicate our method of solving the open-shell RPAE equations of motion in Eq. (1), it suffices to give here only the form of the matrices $U, A$, and $D$ in the approximation that we make the restrictions (i)-(iii) above. From Appendix A, we have

$$
\begin{aligned}
& U_{i \epsilon, j \epsilon^{i}}=\delta\left(\epsilon-\epsilon^{\prime}\right) U_{i j}=\delta\left(\epsilon-\epsilon^{\prime}\right) \delta_{i j} U_{i i}, \\
& A_{i \epsilon, j \epsilon^{\prime}} \cong \delta\left(\epsilon-\epsilon^{\prime}\right) \delta_{i j}\left(\epsilon+E_{i}\right) U_{i i}, \\
& D_{i \epsilon, j \epsilon^{\prime}} \cong \delta_{i j} D_{i \epsilon, i \epsilon^{\prime}} .
\end{aligned}
$$

In Eq. (4), $E_{i}$ is the binding energy in the $i$ th channel. In what follows, then, we consider only interactions within channel $i$. The appropriate multichannel equations, however, may be easily inferred from the equations below provided one diagonalizes the matrix $U_{i j}$ at the outset of a multichannel treatment.

\section{A. General single-channel equations}

Solution of the single-channel open-shell RPAE equations of motion starts by substituting Eqs. (3) -(5) in Eq. (1), noting that all matrix elements are real, and dividing both sides by $U_{i i}$ to obtain the following coupled equations:

$$
\int d \epsilon^{\prime} V_{i \epsilon, i \epsilon^{\prime}} X_{i \epsilon^{\prime}}(\omega)=-\left(\omega+\epsilon+E_{i}\right) Y_{i \epsilon}(\omega),
$$

where

$$
V_{i \epsilon, i \epsilon^{\prime}} \equiv D_{i \epsilon, i \epsilon^{\prime}} / U_{i i}
$$

and the integration over photoelectron energies $\epsilon^{\prime}$ includes a summation over discrete values of $\epsilon^{\prime}$. Equations (6) and (7) can be uncoupled by first solving Eq. (7) for $Y_{i \epsilon}(\omega)$,

$$
Y_{i \epsilon}(\omega)=-\frac{1}{\left(\omega+\epsilon+E_{i}\right)} \int d \epsilon^{\prime} V_{i \epsilon, i \epsilon^{\prime}} X_{i \epsilon^{\prime}}(\omega),
$$

and then substituting Eq. (9) in Eq. (6) to get a single equation for $X_{i \epsilon}(\omega)$,

$$
\int d \epsilon^{\prime} v_{i \epsilon, i \epsilon^{\prime}}(\omega) X_{i \epsilon^{\prime}}(\omega)=\left(\omega-\epsilon-E_{i}\right) X_{i \epsilon}(\omega) .
$$

In Eq. (10) the matrix element $v_{i \epsilon, i \epsilon^{\prime}}(\omega)$ is defined to be

$$
V_{i \epsilon, \boldsymbol{i} \epsilon^{\prime}}(\omega) \equiv-\int d \epsilon^{\prime \prime} V_{i \epsilon, i \epsilon^{\prime \prime}} \frac{1}{\left(\omega+\epsilon^{\prime \prime}+E_{i}\right)} V_{i \epsilon^{\prime \prime}, i \epsilon^{\prime}} .
$$

Note that whereas the matrix element $V_{i \in, i \epsilon^{\prime}}$ denotes the excitation of a pair of electrons out of the open-subshell ground state to the final states $i \epsilon$ and $i \epsilon^{\prime}$, the energy-dependent matrix element $v_{i \epsilon, i \epsilon^{\prime}}(\omega)$ denotes an indirect (i.e., second-order) intrachannel interaction between final states $i \epsilon$ and $i \epsilon^{\prime}$.

Solutions $X_{i \epsilon}(\omega)$ of Eq. (10) may be written quite generally in the following form ${ }^{20(b)}$ :

$$
\begin{aligned}
X_{i \epsilon}(\omega)= & \left\{\left[\odot /\left(\omega-\epsilon-E_{i}\right)\right] K_{i \epsilon, i \bar{\epsilon}}(\omega)\right. \\
& \left.+\delta\left(\omega-\epsilon-E_{i}\right)\right\} B_{i}(\omega) .
\end{aligned}
$$

Here $P$ indicates the Cauchy principal part is to be taken in any integration over the singular denominator. $K(\omega)$ is a reaction matrix, defined by the integral equation obtained upon substituting Eq. (12) in Eq. (10),

$$
\begin{aligned}
K_{i \epsilon, i \bar{\epsilon}}(\omega)=v_{i \epsilon, i \bar{\epsilon}}(\omega)+\mathcal{P} \int & d \epsilon^{\prime} v_{i \epsilon, i \epsilon^{\prime}}(\omega) \\
& \times \frac{1}{\left(\omega-\epsilon^{\prime}-E_{i}\right)} K_{i \epsilon^{\prime}, i \bar{\epsilon}}(\omega) .
\end{aligned}
$$

Note that $X_{i \epsilon}(\omega)$ depends only on the column of the reaction matrix $K(\omega)$ having the index $i \bar{\epsilon}$, where $\bar{\epsilon}$ is the photoelectron energy appropriate to the photon energy $\omega$,

$$
\bar{\epsilon} \equiv \omega-E_{i} .
$$

Lastly, $B_{i}(\omega)$ is a normalization factor determined from the open-shell RPAE normalization condition (cf. Eq. (33) of Ref. 17),

$\int d \epsilon\left[X_{i \epsilon}^{\dagger}(\omega) U_{i i} X_{i \epsilon}\left(\omega^{\prime}\right)-Y_{i \epsilon}^{\dagger}(\omega) U_{i i} Y_{i \epsilon}\left(\omega^{\prime}\right)\right]=\delta\left(\omega-\omega^{\prime}\right)$. 
In Appendix B we show that

$$
B_{i}(\omega)=\left\{U_{i i}\left[1+\pi^{2} K_{i \bar{\epsilon}, i \bar{\epsilon}}^{2}(\omega)\right]\right\}^{-1 / 2} .
$$

Having obtained the coefficient vectors $X_{i \epsilon}(\omega)$ and $Y_{i \epsilon}(\omega)$ for an arbitrary one-electron excitation operator we can now give the open-shell RPAE form for matrix elements of the electric dipole operator in terms of the Coulomb-interaction matrix elements. Denoting the exact ground state by the bra $(g \mid$ and the Hartree-Fock ground state by $\langle g|$ and using similar notation for the final states $i \epsilon$, the reduced matrix elements of the electric dipole operator are given by Ref. 17, Eq. (37), as $^{30}$

$(g\|r\| i \bar{\epsilon})$

$$
\cong\left(U_{i i}\right)^{1 / 2} \int d \epsilon\left\langle g\left\|_{i} r\right\| i \epsilon\right\rangle\left[X_{i \epsilon}(\omega)+Y_{i \epsilon}(\omega)\right]
$$

Substuting Eq. (9) for $Y_{i \epsilon}(\omega)$ in Eq. (17), and then substituting Eq. (12) for $X_{i \epsilon}(\omega)$ and making use of Eqs. (14) and (16), one obtains

$$
\begin{aligned}
(g\|r\| i \bar{\epsilon}) \cong & \left(\langle g\|r\| i \bar{\epsilon}\rangle+\mathcal{P} \int d \epsilon\langle g\|r\| i \epsilon\rangle \frac{1}{\left(\omega-\epsilon-E_{i}\right)} K_{i \epsilon, i \bar{\epsilon}}(\omega)-\int d \epsilon\langle g\|r\| i \epsilon\rangle \frac{1}{\left(\omega+\epsilon+E_{i}\right)} G_{i \epsilon, i \bar{\epsilon}}(\omega)\right) \\
& \times\left\{1+\left[\pi K_{i \bar{\epsilon}, i \epsilon}(\omega)\right]^{2}\right\}^{-1 / 2},
\end{aligned}
$$

where for compactness we have defined

$$
G_{i \epsilon, i \bar{\epsilon}}(\omega) \equiv V_{i \epsilon, i \bar{\epsilon}}+\mathcal{P} \int d \epsilon^{\prime} V_{i \epsilon, i \epsilon^{\prime}} \frac{1}{\left(\omega-\epsilon^{\prime}-E_{i}\right)} K_{i \epsilon^{\prime}, i \bar{\epsilon}}(\omega) .
$$

Equation (18) thus gives the open-shell RPAE reduced electric dipole matrix element as the sum of three terms: the zero-order (i.e., HF) reduced dipole matrix element for photoionization from the ground state to the final state $i \bar{\epsilon}$, the contribution arising from indirect interactions between final states $i \epsilon$ induced by virtual double excitations [cf. Eqs. (11) and (13)], and the contribution arising from the deexcitation of the virtual, doubly excited state $(i \epsilon, i \bar{\epsilon})$ to the singly excited state $i \bar{\epsilon}$ by absorption of the photon $\omega$.

In summary, the reduced dipole matrix elements, from which cross sections and other measurable quantities may be calculated, are given in our one-channel open-shell RPAE treatment by Eq. (18) in terms of zero-order reduced dipole matrix elements and in terms of two energy-dependent matrices $K(\omega)$ and $G(\omega)$. $K(\omega)$ is defined by Eq. (13) in terms of the indirect intrachannel interaction matrix $v(\omega)$, which in turn is defined by Eq. (11) in terms of the zero-order Coulomb matrix elements $V_{i \epsilon, i \epsilon^{\prime}} . G(\omega)$ is also defined in terms of the matrix elements $V_{i \epsilon, i \epsilon^{\prime}}$ in Eq. (19). Any calculation of open-shell RPAE reduceddipole matrix elements thus starts from the computation of the matrix elements $V_{i \epsilon, i \epsilon^{\prime}}$, which are defined by Eq. (8) as the ratio of $D_{i \epsilon, i \epsilon^{\prime}}$ and $U_{i i}$, each of which is given in Appendix A.

B. Calculational details specific to photoionization of atomic chlorine

We have considered the following photoionization reactions in atomic chlorine:

$$
\mathrm{Cl}\left(3 p^{5}\left({ }^{2} P\right)\right)+\hbar \omega \rightarrow \mathrm{Cl}^{+}\left(3 p^{4}\left({ }^{2} S_{c}+1 L_{c}\right)\right)+\epsilon l\left({ }^{2} L\right) .
$$

The allowed values of the ion-term levels ${ }^{2 s_{c}+1} L_{c}$, the photoelectron orbital angular momentum $l$, and the final total orbital angular momentum $L$ for the nine allowed channels $i$ are given in the first three columns of Table I. Column four of Table I specifies the interaction matrix elements $V_{i \epsilon, i \epsilon^{\prime}}$ in each channel $i$ in terms of the Slater integrals $R^{1}$ and $R^{3}$, where in atomic units

$$
\begin{aligned}
R^{k} & \equiv R^{k}\left(3 p 3 p ; \epsilon l \epsilon^{\prime} l\right), \\
& =\int_{0}^{\infty} \int_{0}^{\infty} \frac{r_{<}^{k}}{r_{>}^{k+1}} P_{3 p}\left(r_{1}\right) P_{\epsilon l}\left(r_{1}\right) P_{3 p}\left(r_{2}\right) P_{\epsilon^{\prime} l}\left(r_{2}\right) d r_{1} d r_{2} .
\end{aligned}
$$

TABLE I. Angular momenta and angular momentum factors for the nine allowed photoionization channels for atomic chlorine.

\begin{tabular}{ccccl}
\hline \hline${ }^{2 s_{c}+1} L_{c}$ & $l$ & $L$ & $V_{i \epsilon, i \epsilon^{\prime}}$ & $\alpha$ \\
\hline${ }^{3} P$ & 2 & 2 & $\frac{7}{20} R^{1}+\frac{9}{140} R^{3}$ & -3 \\
${ }^{3} P$ & 2 & 1 & $-\frac{19}{60} R^{1}+\frac{9}{140} R^{3}$ & $+3^{1 / 2}$ \\
${ }^{3} P$ & 0 & 1 & $-\frac{1}{3} R^{1}$ & $+6^{1 / 2}$ \\
${ }^{1} D$ & 2 & 2 & $+\frac{1}{12} R^{1}+\frac{15}{196} R^{3}$ & $-\left(\frac{7}{3}\right)^{1 / 2}$ \\
${ }^{1} D$ & 2 & 1 & $-\frac{1}{4} R^{1}-\frac{3}{28} R^{3}$ & $+3^{1 / 2}$ \\
${ }^{1} D$ & 2 & 0 & $\frac{11}{30} R^{1}+\frac{9}{70} R^{3}$ & $-2 / 3^{1 / 2}$ \\
${ }^{1} D$ & 0 & 2 & $+\frac{1}{3} R^{1}$ & $\left(\frac{10}{3}\right)^{1 / 2}$ \\
${ }^{1} S$ & 2 & 2 & $-\frac{4}{15} R^{1}-\frac{6}{35} R^{3}$ & $-2 / 3^{1 / 2}$ \\
${ }^{1} S$ & 0 & 0 & $-\frac{2}{3} R^{1}$ & $\left(\frac{2}{3}\right)^{1 / 2}$ \\
\hline \hline
\end{tabular}


In Eq. (21) $P_{n l}(r) / r$ is the radial wave function of an $n l$ electron.

The reduced dipole matrix elements are given by

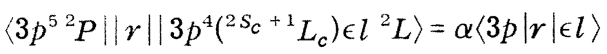

$$
\begin{aligned}
& \equiv-(-1)^{L_{c}} 3(2 \times 5(2 l+1)(2 L+1))^{1 / 2}\left(\begin{array}{c}
11 l \\
000
\end{array}\right)\left\{\begin{array}{ccc}
L_{c} & l & L \\
1 & 1 & 1
\end{array}\right\}\left(3 p^{52} P\left\{\mid 3 p^{4}\left({ }^{2} s_{c}+1 L_{c}\right), 3 p\right)\langle 3 p|r| \epsilon l\rangle,\right.
\end{aligned}
$$

where

$$
\langle 3 p|r| \epsilon l\rangle \equiv \int_{0}^{\infty} P_{3 p}(r) P_{\epsilon l}(r) r d r .
$$

The coefficient $\alpha$ defined in Eq. (22) is given for each of the nine allowed channels in column five of Table I. The channel cross sections for photoionization of atomic chlorine are given in terms of the reduced dipole matrix elements by

$$
\begin{aligned}
& \sigma_{i \epsilon}=\left(0.4484 \times 10^{-18} \mathrm{~cm}^{2}\right)\left(E_{i}+\epsilon\right) \\
& \times\left\langle 3 p^{52} P\|r\| 3 p^{4}\left({ }^{2 s_{c}+1} L_{c}\right) \epsilon l^{2} L\right\rangle^{2},
\end{aligned}
$$

where the binding energies $E_{i}$ were taken from experiment ${ }^{31}$ and averaged over fine-structure levels,

$$
\begin{aligned}
& E_{\left(3_{P}\right)}=0.4768 \text { a.u. }, \\
& E_{\left(1_{D}\right)}=0.5283 \text { a.u. }, \\
& E_{\left(1_{S}\right)}=0.6022 \text { a.u. . }
\end{aligned}
$$

Note that Eq. (24) requires $E_{i}$ and $\epsilon$ to be in atomic units ( 1 a.u. $=27.2108 \mathrm{eV}$ ) and the continuum radial wave functions $P_{\epsilon \ell}(r)$ to be normalized per unit energy (a.u.).

The open-shell RPAE calculations employed a basis set of restricted $\mathrm{HF}$ excited orbitals computed in the $V^{N-1}$ potential ${ }^{28}$ of atomic chlorine minus one $3 p$ electron orbital. A different basis set of continuum orbitals was computed for each allowed channel defined by the quantum numbers in Eq. (2). We shall call this kind of basis the "unrelaxed" $\mathrm{HF}$ basis. The $V^{N-1}$ potential was constructed using the discrete $\mathrm{HF}$ orbitals for atomic chlorine given in the Clementi-Roetti tables. ${ }^{32}$ The first two discrete excited orbitals in each channel were computed using the multiconfiguration $\mathrm{HF}$ computer code of FroeseFisher. ${ }^{33}$ Continuum HF radial wave functions were computed by the method of Dalgarno, Henry, and Stewart ${ }^{34}$ using the computer code of Manson. These continuum HF orbitals correspond to those employed by Kelly and Simons ${ }^{11}$ and to the $\psi^{N(L S)}$ functions of Amus'ya et al. ${ }^{2}$

An alternative HF calculation was also carried out using restricted $\mathrm{HF}$ excited orbitals computed in the $V^{N-1}$ potential constructed from discrete
HF orbitals ${ }^{32}$ appropriate to the alternative term levels of singly ionized chlorine. This second HF basis, called here the "relaxed" HF basis, allows for core relaxation. Because of the slight nonorthogonality of the initial-state (atomic) and final-staie (ionic) discrete $\mathrm{HF}$ orbitals in this second calculation, we did not do an open-shell RPAE calculation using this basis set. The squared overlap integral between initial- and final-state Slater-determinant wave functions is estimated from similar calculation $\mathrm{s}^{35}$ for argon photoionization to be better than $96 \%$. We did not take this nonorthogonality into account and thus our relaxed $\mathrm{HF}$ results are too large by $\lesssim 4 \%$. Our relaxed $\mathrm{HF}$ calculations correspond to similar calculations of Kennedy and Manson. ${ }^{7}$

In solving the open-shell RPAE equations all integrations over photoelectron energy $\epsilon$ imply a summation over discrete energy values. This summation is accomplished by first giving the discrete wave function for the last level $n l$ included in our summation a continuum energy normalization by multiplying it by the factor ${ }^{36}$

$$
\left(\frac{d n}{d \epsilon}\right)_{\epsilon=\epsilon_{n}}^{1 / 2}=\left(-2 \epsilon_{n}\right)^{-3 / 4} \text {. }
$$

The summation over levels $n^{\prime} l$ having $n^{\prime}>n$ is then accomplished by starting the integration over $\epsilon$ at $\epsilon=\epsilon_{n l}$ rather than at threshold.

In all, we employed a basis set in each channel of 17 excited orbitals $\epsilon l$. Two of these were dis crete (either $3 d$ and $4 d$ or $4 s$ and $5 s$ ) and fifteen were in the continuum in the range $0 \leqslant \epsilon \leqslant 8$ a.u. The various integral equations were reduced to a set of linear algebraic equations that were solved by matrix inversion. ${ }^{37}$

Finally, note that all dipole matrix elements were calculated using the dipole length formula. Requiring the HF Hamiltonian to be gauge invariant forces one to choose the length formula for HF calculations. ${ }^{38}$ For RPAE calculations, Amus'ya et $a l .^{2}$ show that length and velocity formulas are equal for closed-shell atoms. Unfortunately, we have not been able to prove this equality in the RPAE for open-shell atoms.

In what follows we present zero-order results using both our relaxed and unrelaxed $\mathrm{HF}$ basis 
sets and open-shell RPAE results starting from the unrelaxed $\mathrm{HF}$ basis.

\section{RESULTS}

A. Partial cross sections

In Tables II-IV we present the individualchannel cross sections for photoionization of atomic chlorine. Each table presents cross sections for channels having in common a particular ion-core term level. The last column of each table gives the sum of these cross sections, i.e., the partial cross section for photoionization of atomic chlorine leaving the ion in a particular term level.

Comparing Tables II-IV with Table I we find that the open-shell RPAE channel cross sections are smaller or larger than the unrelaxed $\mathrm{HF}$ channel cross sections depending on whether the coefficient of the $R^{1}$ Slater integral in the matrix element $V_{i \epsilon, i \epsilon^{\prime}}$ is positive or negative. This is reasonable as seen by inspecting Eqs. (18) and (19), provided that the term in Eq. (18) involving the matrix $K(\omega)$ has only a small influence on the reduced dipole matrix element.

Since $K_{i \epsilon, i \epsilon^{\prime}}(\omega)$ depends on the square of $V_{i \epsilon, i \epsilon^{\prime}}$ while $G_{i \epsilon, i \epsilon^{\prime}}(\omega)$ depends linearly on $V_{i \epsilon, i \epsilon^{\prime}}$, we can check the relative importance of these two matrices by investigating the magnitudes of the matrix elements $V_{i \epsilon, i \epsilon^{\prime}}$. The maximum value of $\left|V_{i \epsilon, i \epsilon^{\prime}}\right|$ for any of the nine channels and for any $\epsilon$ or $\epsilon$ turns out to be 0.35 a.u. for the $\mathrm{Cl}^{+}\left({ }^{1} S\right) \epsilon d\left({ }^{2} D\right)$ channel. This is due to the following circumstances: the $R^{1}$ and $R^{3}$ Slater integrals are relatively large in this channel (e.g., they attain maximum values of 0.94 and 0.55 a.u., respectively), and the coefficients multiplying the $R^{1}$ and $R^{3}$ integrals (cf. Table I) are both sizable and of the same sign in this channel. The other $d$-electron channels have peak values of $V_{i \epsilon, i \epsilon}$ that are $30-90 \%$ of that in the $\mathrm{Cl}^{+}\left({ }^{1} S\right) \epsilon d\left({ }^{2} D\right)$ channel. For these magnitudes of $V_{i \epsilon, i \epsilon^{\prime}}$ we find in all cases the matrix elements of $G(\omega)$ are at least an order of magnitude greater than those of $K(\omega)$. Hence it is the magnitude and sign of $G_{i \epsilon, i \epsilon^{\prime}}(\omega)$ (which is nearly equal to $\left.V_{i \epsilon, i \epsilon^{\prime}}\right)$ that determines the observed behavior of the open-shell RPAE channel cross sections relative to those of the unrelaxed $\mathrm{HF}$-channel cross sections.

From Tables II-IV we see that in each channel the RPAE correction to the HF cross section is frequently greater than $10 \%$. The last column in each table, however, shows that summing the channel cross sections give HF and RPAE partial ionic cross sections (i.e., corresponding to a particular ion term) that are usually well within $10 \%$ of each other. This reduction in the importance of the RPAE corrections for the partial ionic cross sections as compared to the individualchannel cross sections is due to the alternating sign of the RPAE corrections in the individual channels which is discussed above.

TABLE II. Cross sections ${ }^{\text {a }}$ for $\mathrm{Cl}\left(3 p^{5}\left({ }^{2} P\right)\right)+h \nu \rightarrow \mathrm{Cl}^{+}\left(3 p^{4}\left({ }^{3} P\right)\right)+e^{-}\left(\epsilon, l\left({ }^{2} L\right)\right)$.

\begin{tabular}{|c|c|c|c|c|c|c|c|c|c|}
\hline \multirow{2}{*}{$\begin{array}{l}\text { Photon } \\
\text { energy } \\
h \nu \text { (a.u.) }\end{array}$} & \multirow{2}{*}{$\begin{array}{c}\text { Photoelectron } \\
\text { energy } \\
\epsilon \text { (a.u.) }\end{array}$} & \multicolumn{2}{|c|}{$\sigma_{\epsilon d}\left({ }^{2} D\right)^{\mathrm{b}}$} & \multicolumn{2}{|c|}{$\sigma_{\epsilon d}\left({ }^{2} P\right)^{\mathrm{c}}$} & \multicolumn{2}{|c|}{$\sigma_{\epsilon s}\left({ }^{2} P\right)^{\mathrm{d}}$} & \multicolumn{2}{|c|}{$\sigma_{\text {tot }}\left({ }^{3} P\right)^{\mathrm{e}}$} \\
\hline & & $\mathrm{HF}$ & RPA & $\mathrm{HF}$ & RPA & $\mathrm{HF}$ & $\mathrm{RPA}$ & $\mathrm{HF}$ & RPA \\
\hline 0.4768 & 0.000 & 15.37 & 14.69 & 6.85 & 7.59 & 3.00 & 3.10 & 25.22 & 25.38 \\
\hline 0.5268 & 0.050 & 18.57 & 17.53 & 8.19 & 9.07 & 2.61 & 2.69 & 29.37 & 29.29 \\
\hline 0.5768 & 0.100 & 20.40 & 18.99 & 8.62 & 9.51 & 2.27 & 2.35 & 31.29 & 30.85 \\
\hline 0.6518 & 0.175 & 20.89 & 18.93 & 7.77 & 8.53 & 1.87 & 1.93 & 30.53 & 29.39 \\
\hline 0.7268 & 0.250 & 19.09 & 16.72 & 5.92 & 6.47 & 1.55 & 1.61 & 26.56 & 24.80 \\
\hline 0.8268 & 0.350 & 14.36 & 11.98 & 3.45 & 3.78 & 1.23 & 1.28 & 19.04 & 17.04 \\
\hline 0.9768 & 0.500 & 6.95 & 5.39 & 1.25 & 1.41 & 0.893 & 0.931 & 9.09 & 7.73 \\
\hline 1.2268 & 0.750 & 1.23 & 0.817 & 0.161 & 0.202 & 0.569 & 0.595 & 1.96 & 1.61 \\
\hline 1.4768 & 1.00 & 0.089 & 0.032 & 0.003 & 0.008 & 0.395 & 0.413 & 0.487 & 0.453 \\
\hline 1.7268 & 1.25 & 0.014 & 0.034 & 0.016 & 0.011 & 0.291 & 0.304 & 0.321 & 0.349 \\
\hline 1.9768 & 1.50 & 0.114 & 0.139 & 0.051 & 0.045 & 0.224 & 0.233 & 0.389 & 0.417 \\
\hline 2.4768 & 2.00 & 0.267 & 0.270 & 0.098 & 0.096 & 0.146 & 0.151 & 0.511 & 0.517 \\
\hline 3.4768 & 3.00 & 0.319 & 0.301 & 0.109 & 0.113 & 0.076 & 0.078 & 0.504 & 0.492 \\
\hline 5.4768 & 5.00 & 0.216 & 0.201 & 0.067 & 0.071 & 0.030 & 0.030 & 0.313 & 0.302 \\
\hline
\end{tabular}

${ }^{a}$ Cross sections given in units of $10^{-18} \mathrm{~cm}^{2}$.

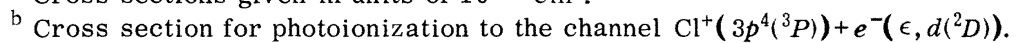

${ }^{c}$ Cross section for photoionization to the channel $\mathrm{Cl}^{+}\left(3 p^{4}\left({ }^{3} P\right)\right)+e^{-}\left(\epsilon, d\left({ }^{2} P\right)\right)$.

${ }^{d}$ Cross section for photoionization to the channel $\mathrm{Cl}^{+}\left(3 p^{4}\left({ }^{3} P\right)\right)+e^{-(}\left(\epsilon, s\left({ }^{2} P\right)\right)$.

${ }^{\mathrm{e}}$ Cross section for photoionization to the ${ }^{3} P$ state of $\mathrm{Cl}^{+}$, equal to the sum of the prior three columns. 


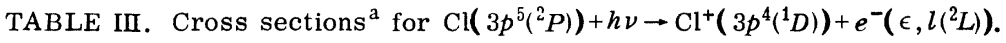

\begin{tabular}{|c|c|c|c|c|c|c|c|c|c|c|c|}
\hline \multirow{2}{*}{$\begin{array}{l}\text { Photon } \\
\text { energy } \\
h \nu \text { (a.u.) }\end{array}$} & \multirow{2}{*}{$\begin{array}{c}\text { Photoelectron } \\
\text { energy } \\
\epsilon \text { (a.u.) }\end{array}$} & \multicolumn{2}{|c|}{$\sigma_{\epsilon d}\left({ }^{2} D\right)^{\mathrm{b}}$} & \multicolumn{2}{|c|}{$\sigma_{\epsilon d}\left({ }^{2} P\right)^{\mathrm{c}}$} & \multicolumn{2}{|c|}{$\sigma_{\epsilon d}\left({ }^{2} S\right)^{\mathrm{d}}$} & \multicolumn{2}{|c|}{$\sigma_{\epsilon s}\left({ }^{2} D\right)^{\mathrm{e}}$} & \multicolumn{2}{|c|}{$\sigma_{\text {tot }}\left({ }^{1} D\right)^{f}$} \\
\hline & & $\mathrm{HF}$ & RPA & $\mathrm{HF}$ & RPA & $\mathrm{HF}$ & RPA & $\mathrm{HF}$ & RPA & $\mathrm{HF}$ & RPA \\
\hline 0.5283 & 0.000 & 9.84 & 9.52 & 11.43 & 13.11 & 4.85 & 4.59 & 1.91 & 1.85 & 28.03 & 29.07 \\
\hline 0.5783 & 0.050 & 10.00 & 9.57 & 12.28 & 13.86 & 5.32 & 4.70 & 1.62 & 1.57 & 29.22 & 29.70 \\
\hline 0.6283 & 0.100 & 8.07 & 7.62 & 10.51 & 11.61 & 4.66 & 3.79 & 1.39 & 1.34 & 24.63 & 24.36 \\
\hline 0.7033 & 0.175 & 4.67 & 4.34 & 6.42 & 6.98 & 2.90 & 2.11 & 1.11 & 1.07 & 15.10 & 14.50 \\
\hline 0.7783 & 0.250 & 2.46 & 2.25 & 3.44 & 3.75 & 1.55 & 1.03 & 0.898 & 0.861 & 8.35 & 7.89 \\
\hline 0.8783 & 0.350 & 1.02 & 0.908 & 1.41 & 1.58 & 0.626 & 0.377 & 0.692 & 0.662 & 3.75 & 3.53 \\
\hline 1.0283 & 0.500 & 0.253 & 0.215 & 0.342 & 0.417 & 0.146 & 0.069 & 0.487 & 0.464 & 1.23 & 1.17 \\
\hline 1.2783 & 0.750 & 0.009 & 0.005 & 0.011 & 0.022 & 0.004 & 0.000 & 0.295 & 0.281 & 0.319 & 0.308 \\
\hline 1.5283 & 1.00 & 0.009 & 0.012 & 0.013 & 0.007 & 0.007 & 0.015 & 0.197 & 0.187 & 0.226 & 0.221 \\
\hline 1.7783 & 1.25 & 0.036 & 0.040 & 0.049 & 0.041 & 0.023 & 0.030 & 0.141 & 0.134 & 0.249 & 0.245 \\
\hline 2.0283 & 1.50 & 0.059 & 0.062 & 0.076 & 0.071 & 0.034 & 0.038 & 0.105 & 0.100 & 0.274 & 0.271 \\
\hline 2.5283 & 2.00 & 0.081 & 0.081 & 0.098 & 0.098 & 0.041 & 0.041 & 0.066 & 0.063 & 0.286 & 0.283 \\
\hline 3.5283 & 3.00 & 0.078 & 0.076 & 0.094 & 0.099 & 0.040 & 0.037 & 0.033 & 0.032 & 0.245 & 0.244 \\
\hline 5.5283 & 5.00 & 0.048 & 0.047 & 0.062 & 0.067 & 0.028 & 0.025 & 0.013 & 0.013 & 0.151 & 0.152 \\
\hline
\end{tabular}

${ }^{a}$ Cross sections given in units of $10^{-18} \mathrm{~cm}^{2}$.

${ }^{\mathrm{b}}$ Cross section for photoionization to the channel $\mathrm{Cl}^{+}\left(3 p^{4}\left({ }^{1} D\right)\right)+e^{-}\left(\epsilon, d\left({ }^{2} D\right)\right)$.

${ }^{c}$ Cross section for photoionization to the channel $\mathrm{Cl}^{+}\left(3 p^{4}\left({ }^{1} D\right)\right)+e^{-}\left(\epsilon, d\left({ }^{2} P\right)\right)$.

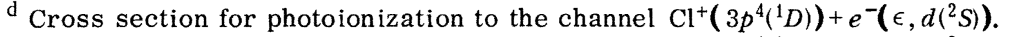

e Cross section for photoionization to the channel $\mathrm{Cl}^{+}\left(3 p^{4}\left({ }^{1} D\right)\right)+e^{-(}\left(\epsilon, s\left({ }^{2} D\right)\right)$.

f Cross section for photoionization to the ${ }^{1} D$ state of $\mathrm{Cl}^{+}$, equal to the sum of the prior four columns.

Figures 1-3 present plots of the partial ionic cross sections in three different approximations. The solid lines are the RPAE cross sections, which improve upon the unrelaxed HF cross sections, represented by the dashed lines. The dotted lines represent the relaxed HF cross sec- tions. The relative behavior of the two types of $\mathrm{HF}$ cross section is similar to their behavior in argon. In argon, the unrelaxed $\mathrm{HF}$ cross section ${ }^{6,11}$ is too high near threshold, while the relaxed $\mathrm{HF}$ cross section ${ }^{7}$ is more nearly the correct height, although shifted toward higher energies. The

TABLE IV. Cross sections a for $\mathrm{Cl}\left(3 p^{5}\left({ }^{2} P\right)\right)+h \nu \rightarrow \mathrm{Cl}^{+}\left(3 p^{4}\left({ }^{1} S\right)\right)+e^{-}\left(\epsilon, l\left({ }^{2} L\right)\right)$.

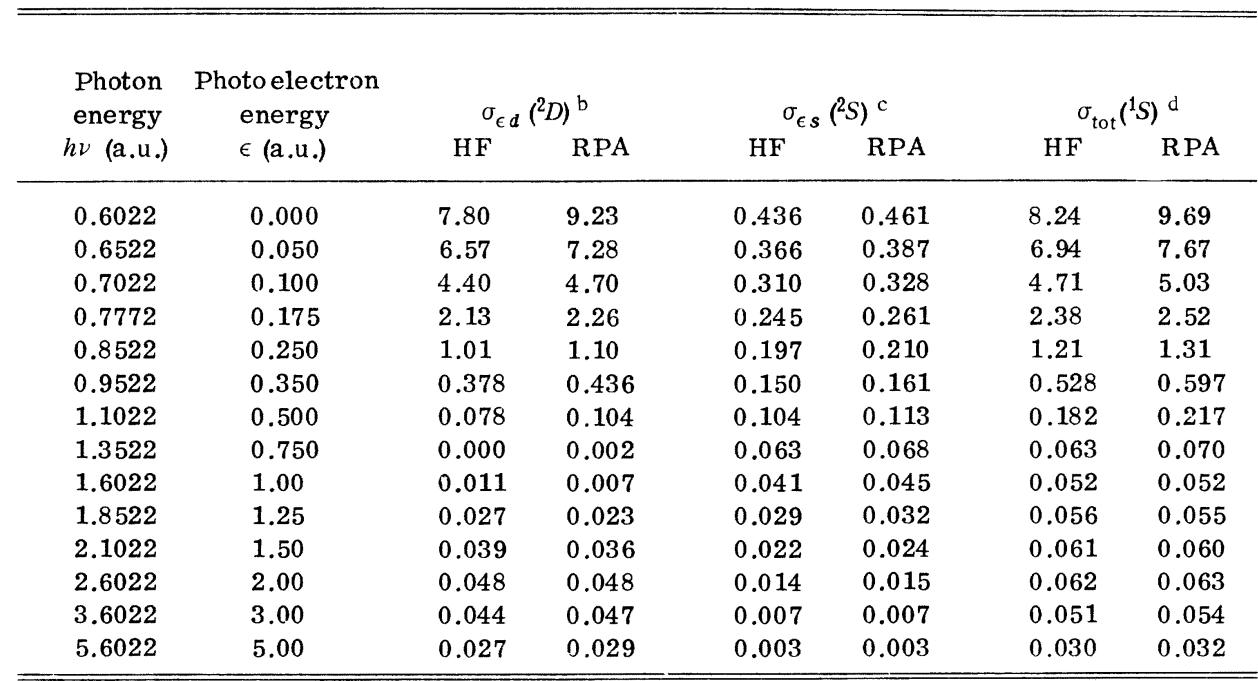

a Cross sections given in units of $10^{-18} \mathrm{~cm}^{2}$.

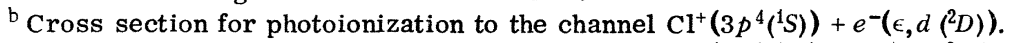

${ }^{c}$ Cross section for photoionization to the channel $\mathrm{Cl}^{+}\left(3 p^{4}\left({ }^{1} S\right)\right)+e^{-}\left(\epsilon, s\left({ }^{2} S\right)\right)$.

${ }^{d}$ Cross section for photoionization to the ${ }^{1} S$ state of $\mathrm{Cl}^{+}$, equal to the sum of the prior two columns. 


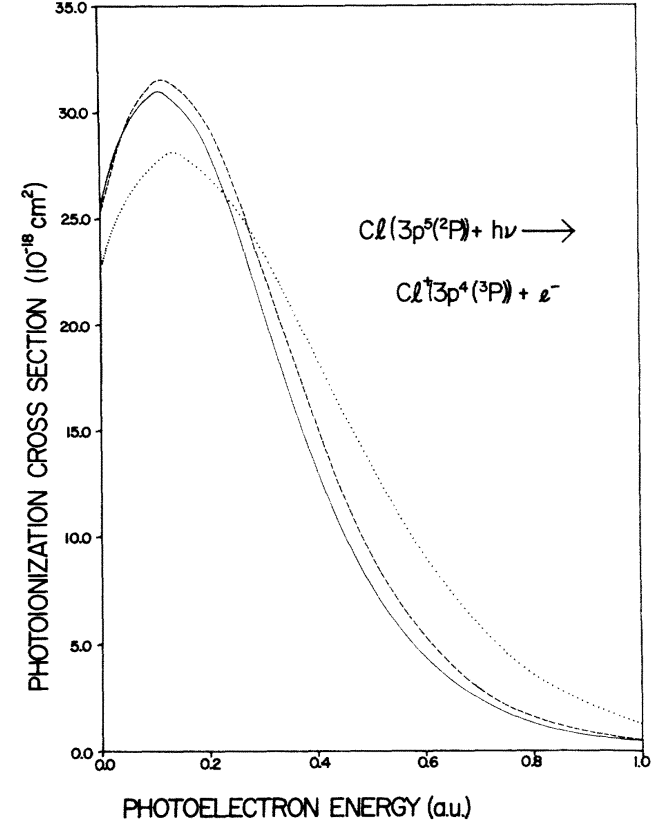

FIG. 1. Partial cross sections for photoionization of atomic chlorine leaving the ion in the ${ }^{3} P$ state. Solid line: open-shell RPAE calculation; dashed line: unrelaxed $\mathrm{HF}$ calculation; dotted line: relaxed $\mathrm{HF}$ calcution.

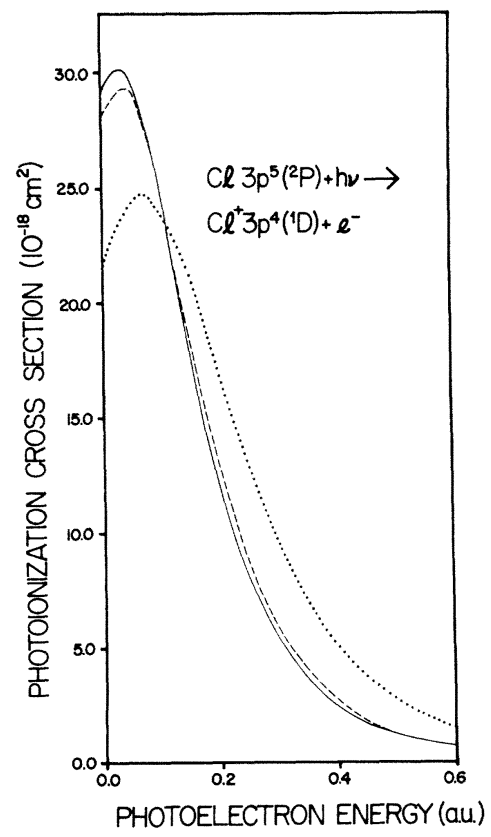

FIG. 2. Partial cross sections for photoionization of atomic chlorine leaving the ion in the ${ }^{1} D$ state. Solid line: open-shell RPAE calculation; dashed line: unrelaxed HF calculation; dotted line: relaxed HF calculation.

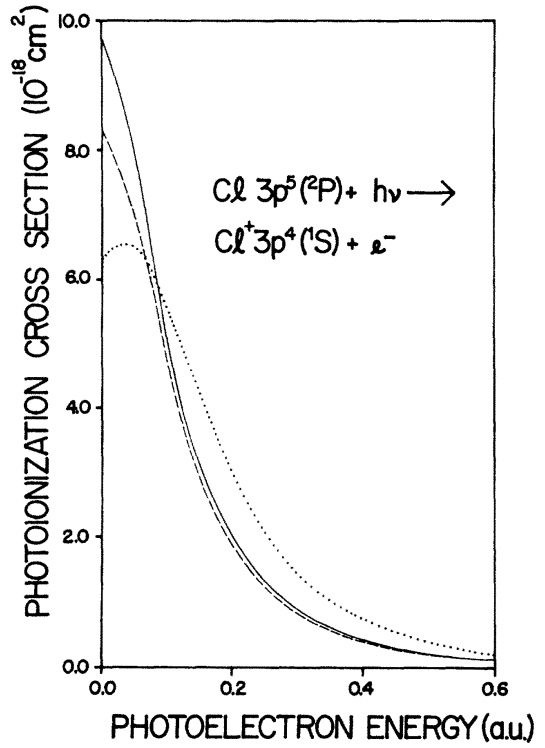

FIG. 3. Partial cross sections for photoionization of atomic chlorine leaving the ion in the ${ }^{1} S$ state. Solid line: open-shell RPAE calculation; dashed line: unrelaxed HF calculation; dotted line: relaxed HF calculation.

RPAE corrections for argon bring the unrelaxed $\mathrm{HF}$ cross section down toward the relaxed $\mathrm{HF}$ cross section near threshold. In chlorine, however, not only does the RPAE cross section show only small deviations from the unrelaxed HF cross section, but in the case of the partial cross sections for the ${ }^{1} D$ and ${ }^{1} S$ ion terms the RPAE corrections to the unrelaxed HF cross section widen the separation from the relaxed $\mathrm{HF}$ cross section near threshold (cf. Figs. 2 and 3). If the relaxed HF cross sections near threshold are as reliable for atomic chlorine as they are for argon, then there would seem to be important relaxation effects in atomic chlorine that are not accounted for by the corrections included in the RPAE. Unfortunately the lack of experimental cross sections for atomic chlorine does not permit a judgment on whether the RPAE or the relaxed $\mathrm{HF}$ cross sections are more accurate near threshold.

\section{B. Photoelectron angular distributions}

We have calculated the photoelectron angular distribution asymmetry parameter $\beta$ for photoionization leading to each of the three ion-term levels. We use the angular momentum transfer formulation for $\beta$ in LS coupling of Dill, Starace, and Manson. ${ }^{39}$ The RPAE corrections to the unrelaxed $H F$ phase shifts are given for each channel $i$ by ${ }^{40}$

$$
\delta_{\mathrm{RPAE}}(\omega)=-\arctan \left[\pi K_{i \bar{\epsilon}, i \bar{\epsilon}}(\omega)\right] .
$$


The maximum value of $o_{\mathrm{RPAF}}(\omega)$ in any channel is $0.1 \mathrm{rad}$. The asymmetry parameters $\beta\left({ }^{3} P\right)$ and $\beta\left({ }^{1} D\right)$ are plotted in Fig. $4 ; \beta\left({ }^{1} S\right)$ is plotted in Fig. 5. The cumulative effect of the differences between HF and RPAE dipole matrix elements and phase shifts is no greater in the case of $\beta$ than it is in the case of the partial ionic cross sections. Note that whereas Figs. 4 and 5 show only the region of photoelectron energies $0 \leqslant \epsilon \leqslant 1.0$ a.u., the near equality of the HF and RPAE results for $\beta$ has been verified up to $\epsilon=5.0 \mathrm{a}$.u.

\section{Total photoionization cross section}

Figure 6 presents our results for the total photoionization cross section as a function of photon energy $h \nu$. The difference between the unrelaxed HF and the RPAE calculations is even smaller here than it is for the partial ionic cross sections. This is because the RPAE corrections have alternate signs in the three ionic cross sections; for the ${ }^{3} \mathrm{P}$ ion term (cf. Table II) the RPAE cross section is smaller than the HF one for $h \nu \geqslant 0.5$ a.u.; for the ${ }^{1} D$ ion term (cf. Table III) the RPAE cross section is larger than the HF one for 0.53 a.u. $\leqslant h \nu \leqslant 0.62$ a.u., but smaller for $h \nu \geqslant 0.62$ a.u.; lastly, for the ${ }^{1} S$ ion term (cf. Table IV) the RPAE cross section is larger than the HF one for $h \nu \geqslant 0.60$ a.u. As is the case for the partial cross sections, here again substantial differences exist between the relaxed and the unrelaxed $\mathrm{HF}$ cross sections that are not corrected by the RPAE.

The crosses in Fig. 6 are Conneely's ${ }^{22,23}$ total

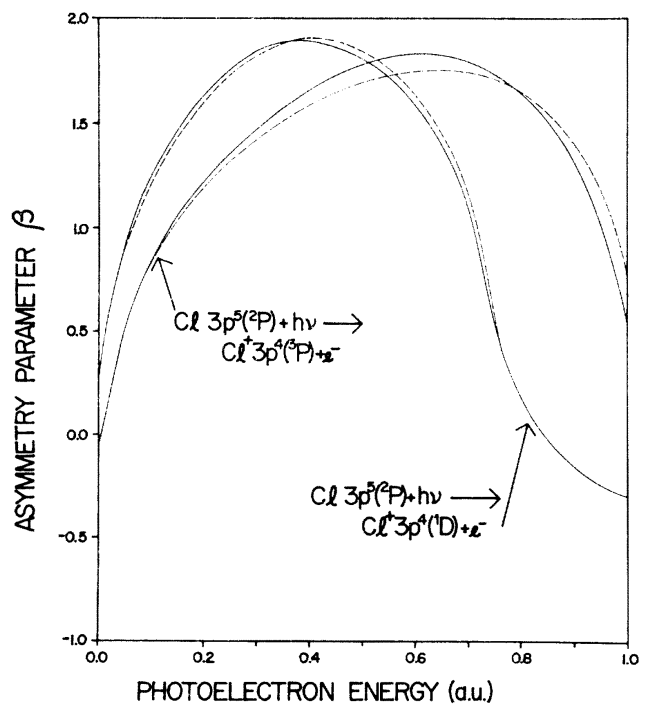

FIG. 4. Photoelectron angular distribution asymmetry parameters $\beta\left({ }^{3} P\right)$ and $\beta\left({ }^{1} D\right)$. Solid line: open-shell RPAE calculation; dashed line: unrelaxed HF calculation.

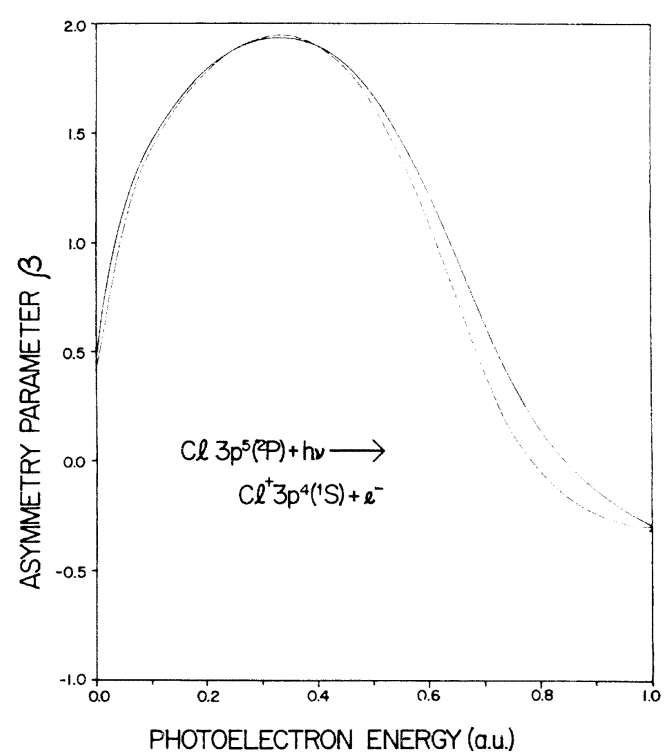

FIG. 5. Photoelectron angular distribution asymmetry parameter $\beta\left({ }^{1} S\right)$. Solid line: open-shell RPAE calculation; dashed line: unrelaxed HF calculation.

close-coupling cross sections for atomic chlorine. Our calculations do not include the interchannel interactions that are included by Conneely. However, as discussed below, there is reason to believe that the large differences between our calculations and those of Conneely are not due to our neglect of interchannel interactions.

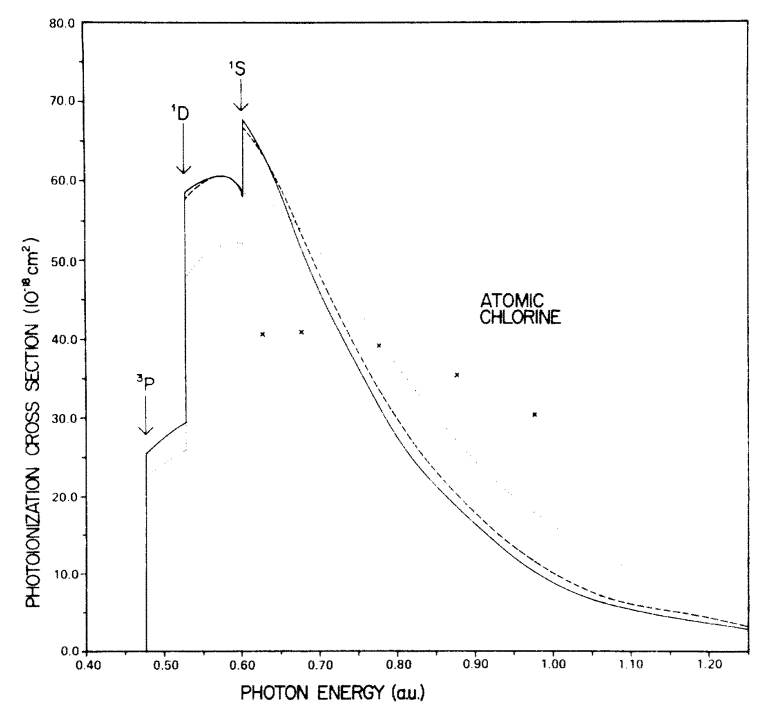

FIG. 6. Total photoionization cross section of atomic chlorine vs photon energy. Ion-term level threshold energies indicated by arrows. Solid line: open-shell RPAE calculation; dashed line: unrelaxed HF calculation; dotted line: relaxed HF calculation. Crosses refer to the close-coupling calculation of Conneely. 


\section{Comparison with calculation of Conneely}

The best comparison between our cross sections and those of Conneely ${ }^{22,23}$ should be for the channel $\mathrm{Cl}\left(3 p^{5}\left({ }^{2} P\right)\right)+h \nu \rightarrow \mathrm{Cl}^{+}\left(3 p^{4}\left({ }^{1} D\right)\right)+e^{-}\left(\epsilon, d\left({ }^{2} S\right)\right)$. This $d$-electron channel can interact only with the $s$ electron channel $\mathrm{Cl}\left(3 p^{5}\left({ }^{2} P\right)\right)+h \nu \rightarrow \mathrm{Cl}^{+}\left(3 p^{4}\left({ }^{1} S\right)\right)$ $+e^{-}\left(\epsilon, s\left({ }^{2} S\right)\right)$, which should probably have only a very small effect on $d$-electron channel. Figure 7 shows our results for this $d$-electron channel and compares them with those of Conneely. In principle, except for the inclusion of the coupling to the $s$-electron channel, Conneely's cross sections for this $d$-electron channel (indicated by crosses) should be identical to our relaxed $\mathrm{HF}$ cross section. For photoelectron energies $\epsilon \geqslant 0.3$ a.u. our relaxed HF cross section is in fact identical to that of Conneely. For $\epsilon \leqslant 0.3$ a.u., however, there are large discrepancies between the two calculations that most likely are not due to our neglect of coupling to the $s$-electron channel.

It was easy for us to check the strength of the $s$ - and $d$-electron channel coupling in argon and at the same time to verify that our relaxed $\mathrm{HF}$ computational methods are working properly. We computed the relaxed $\mathrm{HF}$ cross section for photoionization of argon,

$$
\operatorname{Ar}\left(3 p^{6}\left({ }^{1} S\right)\right)+h \nu \rightarrow \operatorname{Ar}^{+}\left(3 p^{5}\left({ }^{2} P\right)\right)+e^{-}(l=0,2),
$$

ignoring the coupling between the $s$ - and $d$-electron

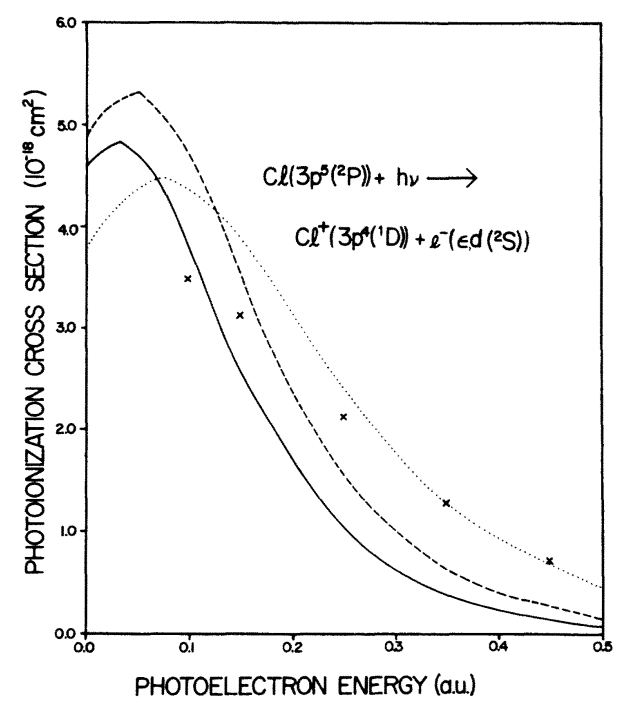

FIG. 7. Partial cross section for photoionization of atomic chlorine leading to ejection of the photoelectron in the channel $\mathrm{Cl}^{+}\left(3 p^{4}\left({ }^{1} D\right)\right)+e^{-}\left(\epsilon, d\left({ }^{2} S\right)\right)$. Solid line: open-shell RPAE calculation; dashed line: unrelaxed HF calculation; dotted line: relaxed HF calculation. Crosses indicate the close-coupling calculation of Conneely. channels. We compared our results to prior calculations of Lipsky ${ }^{9}$ which included (via close coupling) the interaction between the $s$ - and $d$ electron channels. We made sure to use exactly the same $V^{N-1}$ potential and the same binding energies as Lipsky and to take into account effects of nonorthogonality between initial and final basis sets. Thus the only difference between Lipsky's calculation and ours is the interchannel coupling . Yet our results are within 4\% of Lipsky's for all photoelectron energies in the range $0 \leqslant \epsilon \leqslant 2.0 \mathrm{Ry}$.

In summary, the discrepancy between our calculated cross sections for atomic chlorine and those of Conneely may indicate that interchannel coupling is much more important in chlorine than in argon. However, if the interchannel coupling is in fact as weak in chlorine as it is between comparable channels in argon, then the discrepancy must have other, at present unknown, causes. Our agreement to within $4 \%$ with closecoupling calculations of Lipsky ${ }^{9}$ for argon indicates that the discrepancies in chlorine are probably not due to any inefficiencies or inaccuracies in our computer code or method of procedure.

\section{E. Multichannel open-shell RPAE calculation}

Because of the smallness of the intrachannel RPAE corrections to the single-channel cross sections, we have chosen not to proceed at this time to calculate the RPAE interchannel interactions. Using the equations in Appendix A we have, however, calculated the angular factors for these interactions in order to obtain an estimate of their strength. The off-diagonal elements of the $A$ matrix give the direct interaction between photoelectron channels that is included in close-coupling calculations as well as in the RPAE. The off-diagonal elements have a magnitude of $\approx 0.4 \mathrm{R}^{1}\left(3 p \epsilon d ; \epsilon^{\prime} d 3 p\right)$, which is not negligible but at the same time not terribly large. The importance of these interactions can be determined precisely by comparison of the single-channel results we present in this paper with the results of close-coupling calculations using the same binding energies and $V^{N-1}$ potentials. Unfortunately, the comparison between our results and the close-coupling calculations of Conneely is ambiguous at this point, as discussed above.

The off-diagonal elements of the $D$ matrix are included only in the RPAE and represent corrections to the interchannel interactions arising from virtual double excitations. We find that the normalized off-diagonal elements $V_{i \epsilon, i \epsilon^{\prime}}$ defined by

$$
V_{i \epsilon, j \epsilon^{\prime}} \equiv D_{i \epsilon, j \epsilon^{\prime}} /\left(U_{i i} U_{j j}\right)^{1 / 2}
$$

have angular coefficients of 0.1 for the $R^{1}(3 p 3 p$; 
$\left.\epsilon d \epsilon^{\prime} d\right)$ Slater integral. The magnitude of these off-diagonal angular coefficients is thus about one-third the magnitude of those for the diagonal matrix elements (cf. Table I). Since the diagonal matrix elements have only a small effect on the single-channel cross sections, we thus do not expect the off-diagonal interactions $V_{i \epsilon, j \epsilon^{\prime}}$ to alter the cross sections appreciably.

\section{CONCLUSIONS}

We have presented open-shell RPAE calculations of the partial and total photoionization cross sections and of the photoelectron angular distributions for atomic chlorine. Despite the fact that chlorine has only one fewer electron than argon, the RPAE corrections in atomic chlorine have been found to be very much smaller than in argon. The direct reason for this is the smaller magnitude of the matrix elements that take into account the effect of virtual excitations of pairs of $d$ electrons from the ground state. In chlorine, in a typical $d$-electron channel, these matrix elements are $\approx 0.3 R^{1}\left(3 p 3 p ; \epsilon d \epsilon^{\prime} d\right)$, where $R^{1}$ is the largest Slater integral. In argon, by comparison, these matrix elements are $\approx 1.3 R^{1}$. Now in chlorine there are six $d$-electron channels, whereas in argon there is only one. Thus one may argue that the RPAE corrections are, in fact, as strong in chlorine as in argon but are dissipated among six channels. However, the RPAE corrections do not have the same sign in all channels, so that summing the individual channels to obtain the partial ionic cross sections or the total cross section reduces the difference between a RPAE calculation and a HF one.

It might be thought that perhaps only one of the eigenchannels of the photoelectron-ion interaction is strongly modified by the RPAE corrections, but that these modifications are reduced in transforming to the experimentally observed channels given in Table I. To check this we diagonalized the $A$ matrix at a particular photon energy and transformed the $D$ matrix to this new representation. The transformed $D$ matrix had somewhat smaller off-diagonal elements and somewhat larger diagonal elements but no single channel took unto itself all of the RPAE corrections represented in the $D$ matrix. The impossibility of simultaneously diagonalizing both the $A$ and $D$ matrices suggests then that the RPAE corrections are not concentrated in a single channel of some suitable representation.

We are thus forced to conclude that the RPAE may not have as dramatic effects in open-shell atoms as in closed-shell atoms. Since this result in chlorine hinges on geometrical factors rather than dynamical ones (i.e., the Slater integrals are quite large in chlorine-only the angular factors are small), we expect similar results for the other halogen atoms. Alternative open-shell atoms for which the RPAE might introduce stronger correlations would probably have to be of one of the following kinds:

Open-shell atoms having a reduced number of photoionization channels, e.g., as for photoionization from ground or excited states having total orbital angular momentum $L=0$. Unlike the rare gases, however, these spherically symmetric atoms would still have several ionic-term levels to photoionize to and thus there would still be more channels than for the rare gases.

Open-shell atoms having RPAE corrections of the same sign in each photoionization channel, so that the RPAE corrections produce a cumulative effect on the partial and total cross sections. Alternatively, there might be some open-shell atoms for which the RPAE corrections are concentrated in only one channel. However, we know of no systematic way to pinpoint such atoms, if indeed they exist at all.

Lastly, it remains to be determined whether a proper kind of Hartree-Fock calculation (i.e., unrelaxed, relaxed, or perhaps some other) is sufficient to correctly predict open-shell atom cross sections or whether there are other correlation effects, not included in the RPAE, which require a separate treatment. Unfortunately, there is no experimental photoionization data as yet for atomic chlorine (or for other heavy openshell atoms) to answer this question even though the experiment for atomic chlorine is certainly feasible. ${ }^{19}$ For this reason it is desirable to perform other calculations for atomic chlorine, by different methods, particularly because of the unresolved discrepancies between our results and those of Conneely.

\section{ACKNOWLEDGMENTS}

We are most grateful to Professor S. T. Manson for providing us with his continuum HF computer code and for aiding us in the calculation of the asymmetry parameters $\beta$. We wish to thank Professor P. L. Altick and Professor D. J. Rowe for clarifying the procedure for normalizing continuum wave functions in the random-phase approximation. We also wish to thank Professor L. Lipsky and Dr. M. J. Conneely for discussions concerning their close-coupling calculations for argon and chlorine, respectively. Lastly, we are most grateful to Professor U. Fano for helping to suggest explanations for the washing out of RPAE effects in open-shell atoms. 


\section{APPENDIX A: CALCULATION OF THE $U, A$, AND $D$ MATRICES}

We give here the expressions used to calculate the matrix elements $U_{i j}, A_{i j}$, and $D_{i j}$ in the special case that we only consider excitations of a single ground-state subshell $n l_{0}^{N}$. We start in each case from the defining equations of Armstrong, ${ }^{17}$ whose notation may be changed to that of this paper by making the following replacements:

$$
\begin{aligned}
& \bar{S}, \bar{L}, \bar{S}^{\prime}, \bar{L}^{\prime} \rightarrow S_{c}, L_{c}, S_{c}^{\prime}, L_{c}^{\prime}, \\
& S, L, S^{\prime}, L^{\prime} \rightarrow S_{0}, L_{0}, S_{0}, L, \\
& l_{\alpha}, l_{\beta}, l_{m}, l_{n} \rightarrow \epsilon l, \epsilon^{\prime} l^{\prime}, n l_{0}, n l_{0} .
\end{aligned}
$$

The meaning of the symbols on the right-hand side of (A1) may be inferred from Eq. (2) of this paper.

The general formulas of Ref. 17 for the $U, A$, and $D$ matrices all involve the coefficients $N$ and $M$ [defined by Eqs. (17) and (18) of Ref. 17], which are in turn expressed in terms of multiconfiguration coefficients of fractional parentage (MCFP). Because they are ubiquitous, it is convenient to evaluate the coefficients $N$ and $M$ first.

The specific form of $N$ which appears in this particular calculation is

$N\left(S_{c} L_{c}, \gamma \in l, g l_{0}\right)$

$$
=\sum\left(g S _ { 0 } L _ { 0 } \{ | \gamma _ { c } S _ { c } L _ { c } , l _ { 0 } ) \left(\gamma S L\left\{\mid \gamma_{c} S_{c} L_{c}, \epsilon l\right),\right.\right.
$$

where the coefficients of fractional parentage
(CFP) on the right-hand side are the MCFP defined by Armstrong, ${ }^{41}$ and $g, \gamma_{c}$, and $\gamma$ indicate any additional quantum numbers necessary to specify the state of the ground, ionic core, and final configurations, respectively. Use of Eq. (23) of Ref. 41 gives

$$
\begin{aligned}
&\left(g S_{0} L_{0}\left\{\mid \gamma_{c} S_{c} L_{c}, l_{0}\right)\right. \\
&=(N / T)^{1 / 2}\left(l_{0}^{N} g S_{0} L_{0}\left\{\mid l_{0}^{N-1} \gamma_{c} S_{c} L_{c}, l_{0}\right),\right.
\end{aligned}
$$

where the CFP on the right-hand side is the usual one for an $n l_{0}^{N}$ configuration ${ }^{42}$ and $T$ is the total number of electrons in the atom. The value of the second MCFP in Eq. (A2) depends on the specification of $\gamma$. The most obvious and convenient form for $\gamma$ is

$$
\gamma S L \equiv\left(\text { filled subshells) } l_{0}^{N-1} \gamma_{c} S_{c} L_{c}, \epsilon l S L,\right.
$$

in which case

$$
\left(\gamma S L\left\{\mid \gamma_{c} S_{c} L_{c}, \epsilon l\right)=T^{-1 / 2},\right.
$$

and thus

$N\left(S_{c} L_{c}, \gamma \in l, g l_{0}\right)=\left(N^{1 / 2} / T\right)\left(l_{0}^{N} g S_{0} L_{0}\left\{\mid l_{0}^{N-1} \gamma_{c} S_{c} L_{c}, l_{0}\right)\right.$.

In what follows we shall not refer to the filled subshells, as in Eq. (A3b), since they are passive.

The form of $M$ which appears in the calculation of the $D$ matrix is

$M\left(S_{c}^{\prime} L_{c}^{\prime}, \gamma l_{0}, \gamma^{\prime} \epsilon^{\prime} l^{\prime}\right)$

$$
=\sum\left\{\begin{array}{ccc}
\frac{1}{2} & S & S_{c}^{\prime \prime} \\
\frac{1}{2} & S_{0} & S_{c}^{\prime}
\end{array}\right\}\left\{\begin{array}{lll}
l_{0} & L & L_{c}^{\prime \prime} \\
l^{\prime} & L_{0} & L_{c}^{\prime}
\end{array}\right\}(-1)^{S+S_{0}+L+L_{0}+1}\left[S_{c}^{\prime}, L_{c}^{\prime}\right]\left(\gamma S L \{ | \gamma _ { c } ^ { \prime \prime } S _ { c } ^ { \prime \prime } L _ { c } ^ { \prime \prime } , l _ { 0 } ) \left(\gamma^{\prime} S_{0} L_{0}\left\{\mid \gamma_{c}^{\prime \prime} S_{c}^{\prime \prime} L_{c}^{\prime \prime}, \epsilon^{\prime} l^{\prime}\right),\right.\right.
$$

where the summation is over $\gamma_{c}^{\prime \prime}, S_{c}^{\prime \prime}$, and $L_{c}^{\prime \prime}$ and where $\left[S_{c}^{\prime}, L_{c}^{\prime}, \ldots\right] \equiv\left(2 S_{c}^{\prime}+1\right)\left(2 L_{c}^{\prime}+1\right) \ldots$

Evaluation of Eq. (A5) requires specification of $\gamma, \gamma^{\prime}$, and $\gamma_{c}^{\prime \prime}$. We want $\gamma$ to have the form given in Eq. (A3b) and $\gamma^{\prime}$ to be defined by

$$
\gamma^{\prime} S_{0} L_{0} \equiv\left[l_{0}^{N-2} \gamma_{2} S_{2} L_{2},\left(\epsilon l \epsilon^{\prime} l^{\prime}\right) S_{3} L_{3}\right] S_{0} L_{0} .
$$

The choice of $\gamma_{c}^{\prime \prime}$ is limited only by the requirement that it specify all states of the configuration $l_{0}^{N-2} \epsilon l$. The most obvious choice is

$$
\gamma_{c}^{\prime \prime} S_{c}^{\prime \prime} L_{c}^{\prime \prime} \equiv{ }_{1}\left(l_{0}^{N-2} \gamma_{2} S_{2} L_{2}, \epsilon l\right) S_{c}^{\prime \prime} L_{c}^{\prime \prime} .
$$

With these choices of $\gamma, \gamma^{\prime}$ and $\gamma_{c}^{\prime \prime}$ the two MCFP in Eq. (A5) become

$$
\left(\gamma S L\left\{\mid \gamma_{c}^{\prime \prime}, S_{c}^{\prime \prime} L_{c}^{\prime \prime}, l_{0}\right)=(-1)^{S_{c}+S_{c}^{\prime \prime}+L_{c}+L_{c}^{\prime \prime}}\left(\frac{N-1}{T}\right)^{1 / 2}\left[L_{c}, L_{c}^{\prime \prime}, S_{c}, S_{c}^{\prime \prime}\right]^{1 / 2}\left\{\begin{array}{ccc}
l & L & L_{c} \\
l_{0} & L_{2} & L_{c}^{\prime \prime}
\end{array}\right\}\left\{\begin{array}{lll}
\frac{1}{2} & S & S_{c} \\
\frac{1}{2} & S_{2} & S_{c}^{\prime \prime}
\end{array}\right\}\left(l_{0}^{N-1} \gamma_{c} S_{c} L_{c}\left\{\mid l_{0}^{N-2} \gamma_{2} S_{2} L_{2}, l_{0}\right)\right.\right.
$$

and

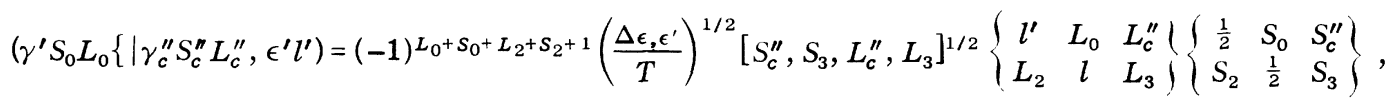


where $\Delta_{\epsilon, \epsilon^{\prime}}=2$ if $\epsilon l=\epsilon^{\prime} l^{\prime}$ and $\Delta_{\epsilon, \epsilon^{\prime}}=1$ otherwise. Inserting Eqs. (A7) and (A8) into Eq. (A5) and summing over $S_{c}^{\prime \prime}$ and $L_{c}^{\prime \prime}$ gives, finally, the desired expression for $M$,

$$
\begin{aligned}
M\left(S_{c}^{\prime} L_{c}^{\prime}, \gamma l_{0}, \gamma^{\prime} \epsilon^{\prime} l^{\prime}\right)= & (-1)^{L_{c}^{\prime}+L_{3}+L_{0^{+}} l_{0}+S_{0^{+}} 1 / 2-S_{c}^{\prime}-S_{3}} T^{-1}\left[(N-1) \Delta_{\epsilon, \epsilon^{\prime}}\right]^{1 / 2}\left[S_{c}^{\prime}, L_{c}^{\prime}\right]\left[L_{c}, S_{c}, L_{3}, S_{3}\right]^{1 / 2} \\
& \times\left\{\begin{array}{lll}
L_{c}^{\prime} & L_{3} & L_{c} \\
L_{2} & l_{0} & L_{0}
\end{array}\right\}\left\{\begin{array}{ccc}
L_{c}^{\prime} & L_{3} & L_{c} \\
l & L & l^{\prime}
\end{array}\right\}\left\{\begin{array}{ccc}
S_{c}^{\prime} & S_{3} & S_{c} \\
S_{2} & \frac{1}{2} & S_{0}
\end{array}\right\}\left\{\begin{array}{ccc}
S_{c}^{\prime} & S_{3} & S_{c} \\
\frac{1}{2} & S & \frac{1}{2}
\end{array}\right\}\left(l_{0}^{N-1} \gamma_{c} S_{c} L_{c}\left\{\mid l_{0}^{N-2} \gamma_{2} S_{2} L_{2}, l_{0}\right) .\right.
\end{aligned}
$$

\section{$U$ matrix}

For the special case of excitations from a partially filled shell to an empty shell, Ref. 17 shows that $U$ is diagonal. The elements of $U$ can be obtained either by combining Eqs. (30) and (32) of Ref. 17 or by substituting Eq. (A4) above in the definition of $U$, given by Eq. (29) of Ref. 17. In either case one obtains

$$
\begin{aligned}
U_{i \epsilon, j \epsilon^{\prime}} & \equiv U\left(S_{c}, L_{c}, \epsilon l, l_{0}, M ; S_{c}^{\prime}, L_{c}^{\prime}, \epsilon^{\prime} l^{\prime}, l_{0}, M\right) \\
& =\delta\left(S_{c}, S_{c}^{\prime}\right) \delta\left(L_{c}, L_{c}^{\prime}\right) \delta\left(l, l^{\prime}\right) \delta\left(\epsilon-\epsilon^{\prime}\right)\left(N / T^{2}\right) \sum_{\gamma_{c}}\left(l_{0}^{N} g S_{0} L_{0}\left\{\mid l_{0}^{N-1} \gamma_{c} S_{c} L_{c}, l_{0}\right)^{2} .\right.
\end{aligned}
$$

\section{$A$ matrix}

Using Eqs. (27) and (29) of Ref. 17 the matrix elements of $A$ can be written

$$
\begin{aligned}
A_{i \epsilon, j \epsilon^{\prime}} & \equiv A\left(S_{c}, L_{c}, \epsilon l, l_{0}, M ; S_{c}^{\prime}, L_{c}^{\prime}, \epsilon^{\prime} l^{\prime}, l_{0}, M\right) \\
& =\sum_{\gamma, \gamma^{\prime}} N\left(S_{c} L_{c}, \gamma \epsilon l, g l_{0}\right)\left(\gamma S L M_{S^{\prime}} M_{L}|H| \gamma^{\prime} S L M_{S} M_{L}\right) N\left(S_{c}^{\prime} L_{c}^{\prime}, \gamma^{\prime} \epsilon^{\prime} l^{\prime}, g l_{0}\right) .
\end{aligned}
$$

Substituting the form of $N$ obtained in Eq. (A4) gives

$$
\begin{aligned}
A_{i \epsilon, j \epsilon^{\prime}}= & \frac{N}{T^{2}} \sum_{\gamma_{c} \gamma_{c}^{\prime}}\left(l _ { 0 } ^ { N } g S _ { 0 } L _ { 0 } \{ | l _ { 0 } ^ { N - 1 } \gamma _ { c } S _ { c } L _ { c } , l _ { 0 } ) \left(l_{0}^{N} g S_{0} L_{0}\left\{\mid l_{0}^{N-1} \gamma_{c}^{\prime} S_{c}^{\prime} L_{c}^{\prime}, l_{0}\right)\right.\right. \\
& \times\left(\left(l_{0}^{N-1} \gamma_{c} S_{c} L_{c}, \epsilon l\right) S L|H|\left(l_{0}^{N-1} \gamma_{c}^{\prime} S_{c}^{\prime} L_{c}^{\prime}, \epsilon^{\prime} l^{\prime}\right) S L\right) .
\end{aligned}
$$

Evaluation of the $A$ matrix is simplified considerably by use of a $V^{N-1}$ potential to calculate excited-state wave functions, since in this case

$$
\begin{array}{r}
\left(\left(l_{0}^{N-1} \gamma_{c} S_{c} L_{c}, \epsilon l\right) S L|H|\left(l_{0}^{N-1} \gamma_{c} S_{c} L_{c}, \epsilon^{\prime} l^{\prime}\right) S L\right) \\
=E\left(\gamma_{c} S_{c} L_{c}, \epsilon l ; S L\right) \delta\left(\epsilon-\epsilon^{\prime}\right) \delta\left(l, l^{\prime}\right),
\end{array}
$$

where $E\left(\gamma_{c} S_{c} L_{c}, \epsilon l ; S L\right)$ is the HF energy of the ${ }^{2 S+1} L$ state corresponding to an excited electron $\epsilon l$ moving in the $V^{N-1}$ potential of the ionic level $\gamma_{c} L_{c} S_{c}$.

Since we have ignored interchannel interactions in this paper, the sum over $\gamma_{c}$ and $\gamma_{c}^{\prime}$ in Eq. (A12) contains only one term, the one having the particular term ${ }^{2 S_{c}+1} L_{c}$ of the ionic configuration $3 p^{4}$ that is under consideration. Thus elements of $A$ diagonal in the ionic core quantum numbers are given by

$$
\begin{aligned}
A\left(S_{c}, L_{c}, \epsilon l, l_{0} ;\right. & \left.S_{c}, L_{c}, \epsilon^{\prime} l^{\prime}, l_{0}\right) \\
& =E\left(S_{c} L_{c}, \epsilon l ; S L\right) \delta\left(\epsilon-\epsilon^{\prime}\right) \\
& \times \delta_{l l^{\prime}}\left(l_{0}^{N} g S_{0} L_{0}\left\{\mid l_{0}^{N-1} \gamma_{c} S_{c} L_{c}, l_{0}\right)^{2} .\right.
\end{aligned}
$$

Equation (A12) shows that matrix elements of $A$ nondiagonal in the ionic core quantum numbers require evaluation of electrostatic matrix elements of the form

$\left(\left(l_{0}^{N-1} \gamma_{c} S_{c} L_{c}, \epsilon l\right) S L\left|\sum_{i>j} \frac{1}{r_{i j}}\right|\left(l_{0}^{N-1} \gamma_{c}^{\prime} S_{c}^{\prime} L_{c}^{\prime}, \epsilon^{\prime} l^{\prime}\right) S L\right)$.

These matrix elements can be easily evaluated by the methods of Ref. 41 or looked up in a number of published works. ${ }^{43,44}$

\section{$D$ matrix}

The $D$ matrix appropriate for this paper is given by Eq. (28) of Ref. 17:

$$
\begin{aligned}
D_{i \epsilon, j \epsilon^{\prime}} \equiv & D\left(S_{c}, L_{c}, \epsilon l, l_{0}, M ; S_{c}^{\prime}, L_{c}^{\prime}, \epsilon^{\prime} l^{\prime}, l_{0}, M\right) \\
=-\frac{1}{2} \sum_{\gamma, \gamma^{\prime}} & {\left[N\left(S_{c} L_{c}, \gamma^{\prime} \epsilon l, g l_{0}\right) M\left(S_{c}^{\prime} L_{c}^{\prime}, \gamma^{\prime} l_{0}, \gamma \epsilon^{\prime} l^{\prime}\right)+N\left(S_{c}^{\prime} L_{c}^{\prime}, \gamma^{\prime} \epsilon^{\prime} l^{\prime}, g l_{0}\right) M\left(S_{c} L_{c}, \gamma^{\prime} l_{0}, \gamma \epsilon l\right)\right] } \\
& \times\left(\gamma S_{0} L_{0} M_{S^{M}} M_{L}|H| g S_{0} L_{0} M_{S} M_{L}\right) .
\end{aligned}
$$


The matrix element of $H$ in Eq. (A15) is given by ${ }^{41}$

$$
\begin{aligned}
\left(\gamma S_{0} L_{0}|H| g S_{0} L_{0}\right)= & \left(\frac{N(N-1)}{\Delta_{\epsilon, \epsilon^{\prime}}}\right)^{1 / 2}(-1)^{L_{3}+1}\left(l_{0}^{N} g S_{0} L_{0}\left\{\mid l_{0}^{N-2} \gamma_{2} S_{2} L_{2}, l_{0}^{2} S_{3} L_{3}\right)\left\{\begin{array}{lll}
l & l^{\prime} & L_{3} \\
l_{0} & l_{0} & k
\end{array}\right\}\right. \\
& \times\left(l_{0}\left\|C^{k}\right\| l\right)\left(l_{0}\left\|C^{k}\right\| l^{\prime}\right) R^{k}\left(l_{0} l_{0} ; \epsilon l \epsilon^{\prime} l^{\prime}\right),
\end{aligned}
$$

where the CFP is a two-body CFP. ${ }^{41,45}$ The sum over $\gamma$ and $\gamma^{\prime}$ in Eq. (A15) is equivalent, in this case, to a sum over $\gamma_{c}, \gamma_{2} S_{2} L_{2}$, and $S_{3} L_{3}$. Substitution of Eqs. (A4), (A9), and (A16) in Eq. (A15) then yields the following expression for the $D$-matrix elements:

$$
\begin{aligned}
& D_{i \epsilon, j \epsilon^{\prime}}=(-1)^{L_{0}-S_{0}+L_{c}^{\prime}+S_{c}^{\prime}+l^{\prime}+S_{3}+1 / 2} \frac{N(N-1)}{\left(2 T^{2}\right)} \\
& \times \sum_{\substack{\gamma_{c} S_{3} L_{3} \\
\gamma_{2} S_{2} L_{2}}}\left[L_{c}, S_{c}, L_{3}, S_{3}, L_{c}, S_{c}\right]^{1 / 2}\left(l _ { 0 } ^ { N } S _ { 0 } L _ { 0 } \{ | l _ { 0 } ^ { N - 1 } \gamma _ { c } S _ { c } L _ { c } , l _ { 0 } ) \left(l_{0}^{N-1} \gamma_{c} S_{c} L_{c}\left\{\mid l_{0}^{N-2} \gamma_{2} S_{2} L_{2}, l_{0}\right)\right.\right. \\
& \times\left(l_{0}^{N} S_{0} L_{0}\left\{\mid l_{0}^{N-2} \gamma_{2} S_{2} L_{2}, l_{0}^{2} S_{3} L_{3}\right)\left\{\begin{array}{ccc}
L_{c} & L_{c}^{\prime} & L_{3} \\
l^{\prime} & l & L
\end{array}\right\}\left\{\begin{array}{ccc}
L_{c} & L_{c}^{\prime} & L_{3} \\
L_{0} & L_{2} & l_{0}
\end{array}\right\}\left\{\begin{array}{ccc}
l & l^{\prime} & L_{3} \\
l_{0} & l_{0} & k
\end{array}\right\}\left\{\begin{array}{ccc}
S_{c} & S_{c}^{\prime} & S_{3} \\
\frac{1}{2} & \frac{1}{2} & S_{0}
\end{array}\right\}\left\{\begin{array}{ccc}
S_{c} & S_{c}^{\prime} & S_{3} \\
S_{0} & S_{2} & \frac{1}{2}
\end{array}\right\}\right. \\
& \times\left(l_{0}\left\|C^{k}\right\| l\right)\left(l_{0}\left\|C^{k}\right\| l^{\prime}\right) R^{k}\left(l_{0} l_{0}, \epsilon l \epsilon^{\prime} l^{\prime}\right)+\left(\text { term with } L_{c} \rightarrow L_{c}^{\prime} \text { and } l \rightarrow l^{\prime}\right) . \\
& \int d \epsilon X_{i \epsilon}^{\dagger}(\omega) U_{i i} X_{i \epsilon}\left(\omega^{\prime}\right)=U_{i i} B_{i}^{\dagger}(\omega) B_{i}\left(\omega^{\prime}\right)\left(\delta\left(\omega-\omega^{\prime}\right)-\frac{1}{\left(\omega-\omega^{\prime}\right)} K_{i \bar{\epsilon}, i \bar{\epsilon}^{\prime}}\left(\omega^{\prime}\right)+K_{i \bar{\epsilon}^{\prime}, i \epsilon}^{\dagger}(\omega) \frac{1}{\left(\omega-\omega^{\prime}\right)}\right. \\
& \left.+\otimes \int d \epsilon K_{i \epsilon, i \bar{\epsilon}}^{\dagger}(\omega) \frac{1}{\left(\omega-\epsilon-E_{i}\right)} \frac{1}{\left(\omega^{\prime}-\epsilon-E_{i}\right)} K_{i \epsilon, i \bar{\epsilon}^{\prime}}\left(\omega^{\prime}\right)\right) .
\end{aligned}
$$

\section{APPENDIX B: NORMALIZATION OF RPAE COEFFICIENTS}

Note that the photoelectron energies in Eq. (B1) having a bar above them are fixed as follows:

$$
\bar{\epsilon} \equiv \omega-E_{i} \text { and } \bar{\epsilon}^{\prime} \equiv \omega^{\prime}-E_{i} \text {. }
$$

Equation (B1) may be simplified by rewriting the product of energy denominators in the last term as follows ${ }^{46}$ :

$$
\left(\omega-\epsilon-E_{i}\right)^{-1}\left(\omega^{\prime}-\epsilon-E_{i}\right)^{-1}=\frac{1}{\left(\omega-\omega^{\prime}\right)}\left(\frac{1}{\left(\omega^{\prime}-\epsilon-E_{i}\right)}-\frac{1}{\left(\omega-\epsilon-E_{i}\right)}\right)+\pi^{2} \delta\left(\omega-\omega^{\prime}\right) \delta\left(\omega-\epsilon-E_{i}\right) .
$$

Substituting Eq. (B3) in Eq. (B1) and rearranging terms gives

$$
\begin{aligned}
\int d \epsilon X_{i \epsilon}^{\dagger}(\omega) U_{i i} X_{i \epsilon}\left(\omega^{\prime}\right)= & U_{i i} B_{i}^{\dagger}(\omega) B_{i}\left(\omega^{\prime}\right) \\
\times & {\left[\delta\left(\omega-\omega^{\prime}\right)\left[1+\pi^{2}\left|K_{i \bar{\epsilon}, i \bar{\epsilon}}(\omega)\right|^{2}\right]\right.} \\
& -\frac{1}{\left(\omega-\omega^{\prime}\right)} \odot \int d \epsilon\left(\delta\left(\omega-\epsilon-E_{i}\right)+K_{i \epsilon, i}^{\dagger}(\omega) \frac{1}{\left(\omega-\epsilon-E_{i}\right)}\right) K_{i \epsilon, i \bar{\epsilon}^{\prime}}\left(\omega^{\prime}\right) \\
& \left.+\frac{1}{\left(\omega-\omega^{\prime}\right)} \odot \int d \epsilon K_{i \epsilon, i \bar{\epsilon}}^{\dagger}(\omega)\left(\delta\left(\omega^{\prime}-\epsilon-E_{i}\right)+\frac{1}{\left(\omega^{\prime}-\epsilon-E_{i}\right)} K_{i \epsilon, i \bar{\epsilon}^{\prime}}\left(\omega^{\prime}\right)\right)\right] .
\end{aligned}
$$

The two integrals in Eq. (B4) cancel identically. This may be verified by using Eq. (13) to substitute in the first integral

$$
\begin{aligned}
K_{i \epsilon, i} \bar{\epsilon}^{\prime}\left(\omega^{\prime}\right)=v_{i \epsilon, i} \bar{\epsilon}^{\prime} & (\omega)+\mathcal{P} \int d \epsilon^{\prime} v_{i \epsilon, i \epsilon^{\prime}}(\omega) \\
& \times \frac{1}{\left(\omega^{\prime}-\epsilon^{\prime}-E_{i}\right)} K_{i \epsilon^{\prime}, i \bar{\epsilon}^{\prime}}\left(\omega^{\prime}\right)
\end{aligned}
$$

and to substitute in the second integral

$$
\begin{aligned}
K_{i \epsilon, i \bar{\epsilon}}^{\dagger}(\omega)=v_{i \epsilon, i \epsilon}^{\dagger}(\omega)+P & \int d \epsilon^{\prime} K_{i \epsilon^{\prime}, i \bar{\epsilon}}^{\dagger}(\omega) \\
& \times \frac{1}{\left(\omega-\epsilon^{\prime}-E_{i}\right)} v_{i \epsilon, i \epsilon^{\prime}}^{\dagger}(\omega) .
\end{aligned}
$$

Exact cancellation follows since $v^{\dagger}(\omega)$ is Her- 
mitian and real [cf. Eq. (11)]. Note that $v(\omega)$ and $v^{\dagger}(\omega)$ both depend on $\omega$ in Eqs. (B5) and (B6) even though the $K$ matrix in Eq. (B5) depends directly on $\omega$ and that in Eq. (B6) on $\omega$. This occurs because in considering the orthonormality properties of states with different energies we must require them to be solutions of the same Hamiltonian, i.e., the one containing $v(\omega)$; otherwise there is no reason for the states to be orthogonal if they have all other quantum numbers the same.

Equation (B4) thus simplifies to

$$
\begin{aligned}
& \int d \epsilon X_{i \epsilon}^{\dagger}(\omega) U_{i i} X_{i \epsilon}\left(\omega^{\prime}\right) \\
&=\left|B_{i}(\omega)\right|^{2} U_{i i}\left[1+\pi^{2}\left|K_{i \bar{\epsilon}, i \bar{\epsilon}}(\omega)\right|^{2}\right] \delta\left(\omega-\omega^{\prime}\right) .
\end{aligned}
$$

A similar treatment of the second term in Eq.
(15) leads to no such simplification. In particular, this second term is completely nonsingular and no $\delta$ function $\delta\left(\omega-\omega^{\prime}\right)$ can be factored out. Equation (15) thus becomes, upon substituting Eq. (B7),

$$
\begin{aligned}
\delta\left(\omega-\omega^{\prime}\right) & =\left|B_{i}(\omega)\right|^{2} U_{i i}\left\lfloor 1+\pi^{2}\left|K_{i \bar{\epsilon}, i \bar{\epsilon}}(\omega)\right|^{2}\right\rfloor \delta\left(\omega-\omega^{\prime}\right) \\
& -\int d \epsilon Y_{i \epsilon}^{\dagger}(\omega) U_{i i} Y_{i \epsilon}\left(\omega^{\prime}\right) .
\end{aligned}
$$

Taking the following integral of both sides,

$$
\lim _{\delta \omega \rightarrow 0} \int_{\omega-\delta \omega}^{\omega+\delta \omega} d \omega^{\prime}
$$

results in

$$
1=\left|B_{i}(\omega)\right|^{2} U_{i i}\left[1+\pi^{2}\left|K_{i \bar{\epsilon}, i \bar{\epsilon}}(\omega)\right|^{2}\right],
$$

which proves Eq. (16) in the text.
*Supported by National Aeronautics and Space Administration Grant No. NGR 28-004-021 and U. S. Energy Research and Development Administration Grant No. $\mathrm{E}(11-1)-2892$.

$\dagger$ Alfred P. Sloan Fellow.

\$Supported in part by the National Science Foundation.

${ }^{1}$ R. D. Hudson and L. J. Kieffer, At. Data 2, 205 (1971).

${ }^{2}$ (a) M. Ya. Amus'ya, N. A. Cherepkov, and L. V. Chernysheva, Zh. Eksp. Teor. Fiz. 60, 160 (1971) [Sov. Phys.-JETP 33, 90 (1971)]; (b) M. Ya. Amusia, Proceedings of the Eighth International Conference on the Physics of Electronic and Atomic Collisions, Belgrade, 1973, Invited Lectures, edited by B. C. Cobic and M. V. Kurepa (Institute of Physics, Belgrade, 1973), p. 172.

${ }^{3}$ G. Wendin, J. Phys. B $\underline{4}, 1080$ (1971); 모 110 (1972); 6, 42 (1973).

${ }^{4}$ C. D. Lin, (a) Phys. Rev. A $\underline{9}, 171$ (1974); (b) $\underline{9}, 181$ (1974).

${ }^{5}$ A. F. Starace, J. Phys. B 7, 14 (1974).

${ }^{6} \mathrm{M}$. Ya. Amus'ya, N. A. Cherepkov, L. V. Chernysheva, and S. I. Sheftel, Zh. Eksp. Teor. Fiz. 56, 1897 (1969) [Sov. Phys._JE'TP 29, 1018 (1969)].

${ }^{7}$ D. J. Kennedy and S. T. Manson, Phys. Rev. A $\underline{5}, 227$ (1972).

${ }^{8}$ A. F. Starace, Phys. Rev. A 2 , 118 (1970).

${ }^{9}$ L. Lipsky and J. W. Cooper (unpublished). See Fig. 22 of Ref. 20 (a).

${ }^{10}$ J. A. R. Samson, J. Opt. Soc. Am. 55, 935 (1965).

${ }^{11}$ H. P. Kelly and R. L. Simons, Phys. Rev. Lett. $\underline{30}, 529$ (1973).

${ }^{12}$ P. G. Burke and K. T. Taylor, J. Phys. B $\underline{8}, 2620$ (1975).

${ }^{13} \mathrm{G}$. Wendin, Phys. Lett. 51A, 291 (1975).

${ }^{14}$ P. L. Altick and A. E. Glassgold, Phys. Rev. 133, A632 (1964).

${ }^{15}$ N. A. Cherepkov, L. V. Chernysheva, V. Radojevic, and I. Pavlin, Can. J. Phys. 52, 349 (1974).

${ }^{16}$ E. Dalgaard, J. Phys. B 8,695 (1975).

${ }^{17}$ L. Armstrong, Jr., J. Phys. B 7, 2320 (1974).
${ }^{18} \mathrm{D}$. J. Rowe and C. Ngo-Trong, Rev. Mod. Phys. 47, 471 (1975).

${ }^{19} \mathrm{~J}$. Berkowitz (private communication); D. Spence (private communication)

${ }^{20}$ (a) U. Fano and J. W. Cooper, Rev. Mod. Phys. 40, 441 (1968); (b) U. Fano, Phys. Rev. 124, 1866 (1961).

${ }^{21}$ E. J. McGuire, Phys. Rev. 175, 20 (1968).

${ }^{22}$ M. J. Conneely, Ph.D. thesis (London University, 1969) (unpublished).

${ }^{23}$ M. J. Conneely, K. Smith, and L. Lipsky, J. Phys. B $\underline{3}, 493$ (1970).

${ }^{24} \mathrm{G}$. E. Brown, Unified Theory of Nuclear Models and Forces (North-Holland, Amsterdam, 1967), 2nd ed., Chap. V.

${ }^{25}$ D. J. Rowe, Rev. Mod. Phys. $\underline{40}, 153$ (1968).

${ }^{26}$ A. L. Fetter and J. D. Walecka, Quantum Theory of Many-Particle Systems (McGraw-Hill, New York, 1971), Chap. 15.

${ }^{27} \mathrm{M}$. Ya. Amus'ya, V. K. Ivanov, N. A. Cherepkov, and L. V. Chernysheva, Zh. Eksp. Teor. Fiz. 66, 1537 (1974) [Sov. Phys._JETP 39, 752 (1975)]; Ref. 4 (a).

${ }^{28}$ H. P. Kelly, Adv. Chem. Phys. 14, 129 (1969).

${ }^{29}$ R. J. W. Henry and L. Lipsky, Phys. Rev. 153, 51 (1967).

${ }^{30}$ For our purposes the summation in Eq. (37) of Ref. 17 is only over configurations $\gamma^{\prime}$ and not over $\bar{S}, \bar{L}, l_{\alpha}$, and $l_{m}$, because of the restrictions we have described in Sec. II of this paper.

${ }^{31} \mathrm{C}$. E. Moore, Ionization Potentials and Ionization Limits Derived from the Analyses of Optical Spectra (U.S. GPO, Washington, D.C., 1970).

${ }^{32}$ E. Clementi and C. Roetti, At. Data Nucl. Data Tables 14,177 (1974).

${ }^{33} \mathrm{C}$. Froese-Fischer, Comput. Phys. Commun. 4, 107 (1972).

${ }^{34}$ A. Dalgarno, R. J. W. Henry, and A. L. Stewart, Planet. Space Sci. 12, 235 (1964).

${ }^{35} \mathrm{~L}$. Lipsky (private communication).

${ }^{36}$ Ref. 20 (a), Sec. 2.4 .

${ }^{37}$ P. L. Altick and E. N. Moore, Phys. Rev. 147, 59 
(1966).

${ }^{38}$ A. F. Starace, Phys. Rev. A 3, 1242 (1971); 8, 1141 (1973); I. P. Grant and A. F. Starace, J. Phys. B 8, 1999 (1975).

${ }^{39}$ D. Dill, A. F. Starace, and S. T. Manson, Phys. Rev. A 11,1596 (1975).

${ }^{40}$ Ref. 20 (a), Eq. (6.23).

${ }^{41}$ L. Armstrong, Jr., Phys. Rev. 172, 18 (1968).

${ }^{42} \mathrm{C}$. W. Nielson and G. F. Koster, Spectroscopic Coefficients for the $p^{N}, d^{N}$, and $f^{N}$ Configurations (MIT, Cambridge, Mass., 1963).
${ }^{43}$ B. G. Wybourne, Spectroscopic Properties of the Rare Earths (Interscience, New York, 1965), Sec. 2-10.

${ }^{44}$ I. I. Sobel'man, An Introduction to the Theory of Atomic Spectra (Pergamon, Oxford, 1972), Sec. 18.2.

${ }^{45} \mathrm{~V}$. L. Donlan, Tables of Two-Particle Fractional Parentage Coefficients for the $p^{N}, d^{N}$, and $f^{N}$ Configurations, U.S. Air Force Materials Laboratory, Wright-Patterson Air Force Base, Ohio 45433, Report No. AFML-TR-70-249 (1970) (unpublished).

${ }^{46}$ Ref. 20 (b), Eq. (11). 